\title{
Strategies of molecular imprinting-based fluorescence sensors for chemical and biological analysis
}

\author{
Qian Yang ${ }^{\mathrm{a}, \mathrm{b}}$, Jinhua $\mathrm{Li}^{\mathrm{b}}$, Xiaoyan Wang ${ }^{\mathrm{b}, \mathrm{c}}$, Hailong Peng ${ }^{\mathrm{a}}$, Hua Xiong ${ }^{\mathrm{a}, *}$, Lingxin Chen ${ }^{\mathrm{b}, *}$ \\ a State Key Laboratory of Food Science and Technology, Nanchang University, Nanchang 330047, China \\ ${ }^{\mathrm{b}}$ Key Laboratory of Coastal Environmental Processes and Ecological Remediation; Research Centre for Coastal Environmental Engineering and Technology, Yantai Institute \\ of Coastal Zone Research, Chinese Academy of Sciences, Yantai 264003, China \\ ' School of Pharmacy, Binzhou Medical University, Yantai 264003, China
}

\section{A R T I C L E I N F O}

\section{Keywords:}

Molecular imprinting

Sensor

Fluorescent detection

Construction strategy

Microdevice

\begin{abstract}
A B S T R A C T
One pressing concern today is to construct sensors that can withstand various disturbances for highly selective and sensitive detecting trace analytes in complicated samples. Molecularly imprinted polymers (MIPs) with tailor-made binding sites are preferred to be recognition elements in sensors for effective targets detection, and fluorescence measurement assists in highly sensitive detection and user-friendly control. Accordingly, molecular imprinting-based fluorescence sensors (MI-FL sensors) have attracted great research interest in many fields such as chemical and biological analysis. Herein, we comprehensively review the recent advances in MI-FL sensors construction and applications, giving insights on sensing principles and signal transduction mechanisms, focusing on general construction strategies for intrinsically fluorescent or nonfluorescent analytes and improvement strategies in sensing performance, particularly in sensitivity. Construction strategies are well overviewed, mainly including the traditional indirect methods of competitive binding against pre-bound fluorescent indicators, employment of fluorescent functional monomers and embedding of fluorescence substances, and novel rational designs of hierarchical architecture (core-shell/hollow and mesoporous structures), post-imprinting modification, and ratiometric fluorescence detection. Furthermore, MI-FL sensor based microdevices are discussed, involving micromotors, test strips and microfluidics, which are more portable for rapid point-of-care detection and in-field diagnosing. Finally, the current challenges and future perspectives of MI-FL sensors are proposed.
\end{abstract}

\section{Introduction}

One pressing concern in environmental monitoring and food security is hazardous materials detection, involving toxic metals, environmental estrogens, drug residues, mycotoxin and so forth. These contaminants pose a great public health threat when people expose themselves to them in the short or long term, directly or indirectly. Likewise, concentration measurement of medicines and biomolecules including vitamin, glucose, neurotransmitter, protein, etc. makes great sense in clinical diagnostics, therapeutics and proteomics research. Detection of illicit substances such as explosives plays a critical role in anti-terrorism and homeland security as well. The ultralow content of these analytes in complex samples has motivated the development of sensitive and selective detection methods, and therefore, high-performance liquid chromatography (HPLC) and gas chromatography (GC) combined with mass spectrometry (MS) or other advanced technologies are preferred; however, the sophisticated sample pretreatment, costly laboratory equipment and well-trained personnel are luxuries in less developed laboratories or sampling points. Hence, highly selective and sensitive as well as economical and user-friendly detection strategies are still in urgent need.

Molecularly imprinted polymers (MIPs), mimicking the behavior of antibodies, are promising for selectively recognizing targets through effectively discriminating against coexisting analogues owing to their tailor-made binding sites. These binding sites are usually created by copolymerizing the functional monomer and cross-linker in the presence of template molecules, with subsequent removal of templates, and thereby are complementary to the target analytes in shape, size and functional groups (Chen et al., 2011). Additionally, compared with the natural counterparts, MIPs can withstand harsher conditions including high temperature and pressure, extreme acid/base and organic solvents. For these unique features of structure predictability, recognition specificity and application universality, MIPs have received widespread attention and played an important role in the analytical studies,

\footnotetext{
* Corresponding authors.

E-mail addresses: huaxiong100@126.com (H. Xiong), lxchen@yic.ac.cn (L. Chen).
} 
particularly in sample pretreatment, chromatographic packing materials and chemo/biosensing (Chen et al., 2016).

In the 1980s, Andersson et al. (1988) pioneered the first MIPs-based sensor for vitamin $\mathrm{K}_{1}$ binding monitoring via optical surface ellipsometry. Since then, MIPs have made considerable progress in the sensing field, combining with electrochemical (Yang et al., 2018; El Gohary et al., 2015; Udomsap et al., 2014), piezoelectric (EL-Sharif et al., 2015) and optical (Li et al., 2011a; Yang et al., 2017a) transduction mechanisms to transduce the specific binding events into measurable signals. Specifically, MIPs-based fluorescence sensors have been brought to the front of the optical sensors for their super sensitivity, particularly when they are compared with other light-based methods such as absorbance: the fluorescence emission signal is collected under a blank background and proportional to the analyte concentration. Its sensitivity could be enhanced by enlarging incident intensity or improving photomultiplier and amplification system when the target analyte is at an extremely low concentration. However, absorbance $(A)$ is defined as the logarithm of the ratio of light intensities measured before $\left(I_{0}\right)$ and after $(I)$ passing through the sample $\left(A=\lg \left(I_{0} / I\right)\right)$. When the analyte is at trace concentration, the difference between incident intensity $\left(I_{0}\right)$ and emergent light $(I)$ is too small to generate measurable absorbance against the blank signal. Regrettably, enlarging incident intensity becomes useless because the emergent light is brightened concurrently (Martinez-Manez and Sancenón, 2003).

As illustrated in Fig. 1, through introducing fluorescence source whose fluorescence characteristics changed upon targets recognition in the common MIPs construction process, the proposed molecular imprinting-based fluorescence sensors (MI-FL sensors) can tactfully cover the demand of highly selective and sensitive readout of the targets contents in complicated samples, through exhibiting specific fluorescence characteristics or even visible fluorescence color change upon specific analyte binding, without further time-consuming templates extracting from the MIPs network (Liu et al., 2013).

In this review, we comprehensively cover the MI-FL sensors in sensing principles, signal transduction mechanisms (from binding events to fluorescence signals), general construction procedures for (non)fluorescent targets and ingenious strategies for sensing performance improvement. Furthermore, for better application in point-ofcare detection, the sensors are constructed on the microdevice platforms of micromotors, test strips and microfluidics. Finally, we propose the current challenges and future perspectives for accelerating the development of MI-FL sensors and extending their chemical and biological applications.

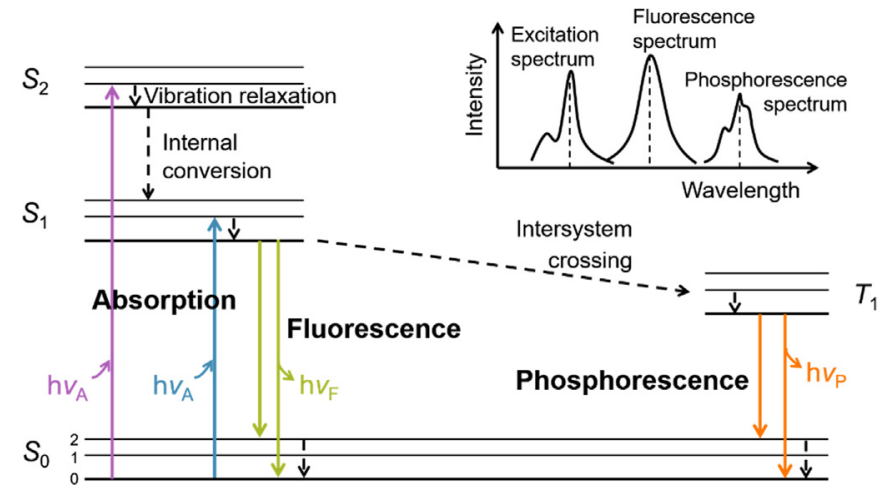

Fig. 2. Jablonski diagram for fluorescence phenomenon. (Reproduced from Lakowicz, 2006).

\section{Fundamentals for MI-FL sensors}

\subsection{Sensing principle}

The formation of fluorescence can be illustrated by a Jablonski diagram (Fig. 2): a loosely held electron at ground state $\left(S_{0}\right)$ would be promoted to the excited state $\left(S_{1}\right.$ or $S_{2}$ ) by absorbing adequate energy, and after some nonradiative transition of vibrational relaxation and/or internal conversion, the excited electron would return to $S_{0}$ from the minimum vibration level of $S_{1}$, accompanying fluorescence emission centered at a larger wavelength than that of the excitation spectrum (called Stokes' Shift) (Lakowicz, 2006). In the target analytes detection, the selective interaction between MI-FL sensors and analytes can affect the fluorescence characteristic of sensors to output specific fluorescence signal including fluorescence brightness (quenching or enhancement), characteristic wavelength (blue or redshift), anisotropy and lifetime through different signal transduction mechanisms. Additionally, these signal changes are dosage-sensitive for providing rough quantitative estimation for the analyte concentration.

Specifically, fluorescence intensity changes are usually observed in MI-FL sensors upon analytes recognition, particularly the fluorescence quenching (defined as "turn-off" mode) (Liu et al., 2016; Xu et al., 2013; Yan et al., 2017). For example, in the 2,4,6-trinitrotoluene (TNT) sensing procedure by quantum dots (QDs)-trinitrophenol-imprinted polymers composites, primary amino groups of QDs could form a Meisenheimer complex with TNT that had a strong electron-withdrawing ability, and then the excited electron transferred to the complex and thus caused quenching of the QDs (Xu et al., 2013). The

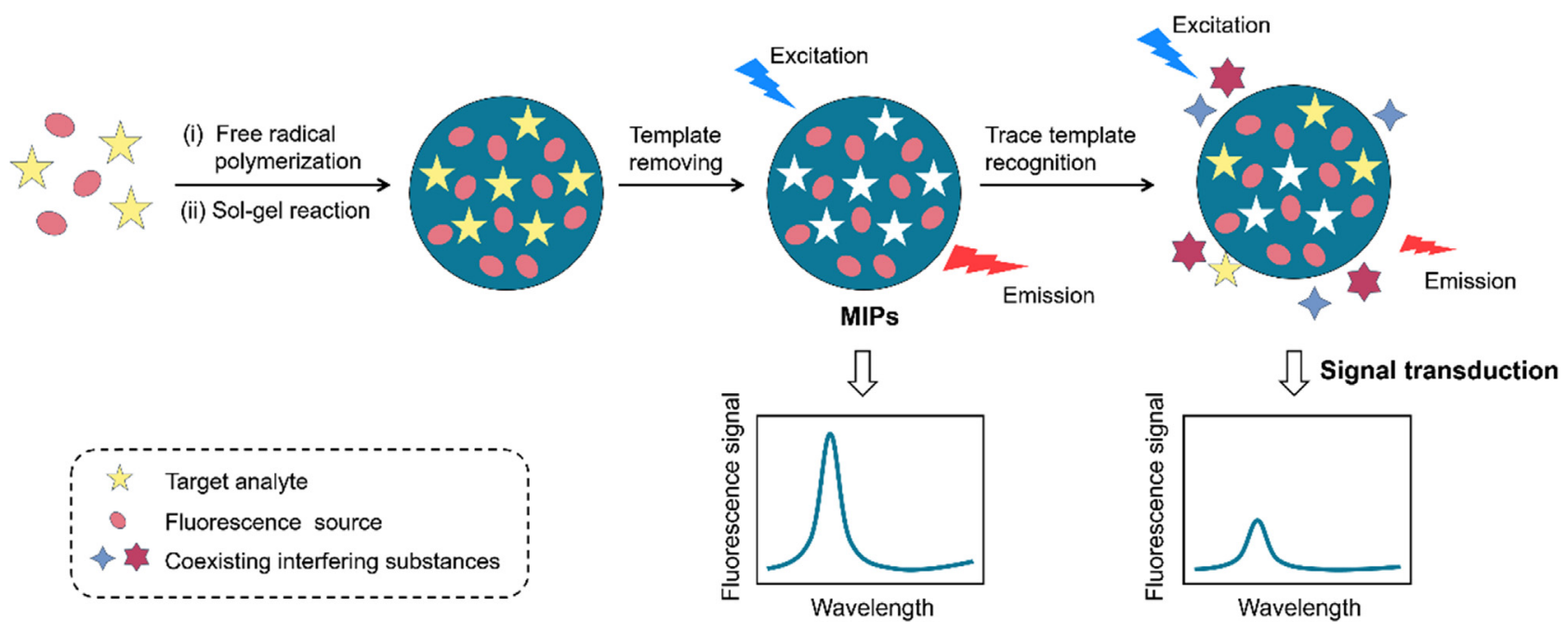

Fig. 1. Schematic overview of molecular imprinting-based fluorescence sensors (MI-FL sensors) construction. 
fluorescence-quenching fractions of the sensor linearly depended on the concentration of TNT, allowing the TNT to be detected optically.

Generally, fluorescence quenching divides into static quenching, dynamic quenching and a combination of the two. Static quenching refers to complex formation between the quencher and the fluorophore at ground state, making no impact to the excited fluorophore and its lifetime. Hence, $\tau_{0} / \tau=1$, where $\tau_{0}$ and $\tau$ are the lifetimes of the fluorophore in the absence and presence of the quencher, respectively. Dynamic quenching means a diffusive encounter between the quencher and the excited fluorophore so that the average fluorescence lifetime decreases, expressed as $\tau_{0} / \tau=F_{0} / F \neq 1$, where $F_{0}$ and $F$ are the fluorescence intensities in the absence and presence of the quencher, respectively. Apart from measuring the fluorescence lifetime change upon quencher, observing their dependence on temperature can also distinguish static and dynamic quenching: increasing temperature causes viscosity decrease of the solution, accelerating diffusion and collisional quenching; oppositely, high temperature would decrease the static quenching constant by declining the stability of complex (Lakowicz, 2006). Additionally, comparing the absorption spectrum can also allow for judging them: ground-state complex generation in static quenching can change the absorption spectrum, but not dynamic quenching.

However, no matter what type the fluorescence quenching is, the change of fluorescence intensity $\left(F_{0} / F\right)$ in a single static or dynamic quenching process is linearly related to the quenching reagent concentration, which can be described by the Stern-Volmer equation as $F_{0} /$ $F=1+K_{\mathrm{S}}[Q]=1+K_{\mathrm{D}}[Q]$, where $K_{\mathrm{S}}$ and $K_{\mathrm{D}}$ are the Stern-Volmer constants for the static and dynamic quenching process, respectively; [Q] is the quencher concentration (Sun et al., 2015). The value of $K_{\mathrm{S}}$ is usually higher than $100 \mathrm{~L} \mathrm{~mol}^{-1}$, and $K_{\mathrm{D}}$ is often less than $100 \mathrm{~L} \mathrm{~mol}^{-1}$. In addition, the Stern-Volmer curve is nonlinear and has an upward movement when the quenching process is a combination of static and dynamic modes (Liu et al., 2014a; Ware, 1962).

As another form of fluorescence intensity changes, fluorescence enhancement (defined as "turn-on" mode) is more attractive, where the specific target recognition turns the "dark" MI-FL sensor into a fluorescent one, or causes the initial emission peak blue/redshift from $\lambda_{\mathrm{i}}$ to $\lambda_{\mathrm{f}}$, and the fluorescence intensity monitored at $\lambda_{\mathrm{f}}$ is read as enhancement (Fig. 3) (Shustova et al., 2013). Stronger sensitivity is more easily achieved in "turn-on" mode due to its lower optical background and higher signal-to-noise ratio, with a more obvious visual effect because the appearance of a bright signal on a completely dark ground is more easily observed than the dimming of an already bright signal (Sun et al., 2015). However, fluorescence enhancement is less applied in MI-FL sensors (Chen et al., 2017a; Li et al., 2015a; Rouhani and Nahavandifard, 2014). For example, Li et al. (2015a) proposed a turnon sensor for tyrosine phosphopeptide (pTyr) detection by epitope imprinting on the surface of silica-capped CdTe QDs. The specific rebinding of phenylphosphonic acid (the epitope) and pTyr passivated trap states in the surface of CdTe QDs, enhanced the fluorescence intensity and therefore improved the detection sensitivity.

\subsection{Signal transduction mechanism}

Generally, MI-FL sensors have both recognition and fluorescence output moieties, and the recognition events can be expressed by fluorescence signals via a specific transduction mechanism, including photoinduced electron transfer (PET), fluorescence resonance energy transfer (FRET) and some others. Below, we would introduce PET and FRET in detail, with fluorophores standing for the fluorescence output moieties.

\subsubsection{Photo-induced electron transfer}

Photo-induced electron transfer (PET) mechanism has been widely used in MI-FL sensors, with the excited fluorophore acting as either an electron donor or acceptor (Lakowicz, 2006). Herein, we take electronacceptor-based fluorophore as an example. Naturally, an excited electron of a fluorophore would go back to the highest occupied molecular orbital (HOMO) from the lowest unoccupied molecular orbital (LUMO) with fluorescence emitting. However, if an orbital occupied by lone pair electrons has energy between those of the fluorophore's HOMO and LUMO, one electron will transfer from this full orbital to the fluorophore's HOMO, filling the vacancy that originally belongs to the excited electron and therefore resulting in the fluorescence quenching (Kim and Quang, 2007).

The specific orbital can come from the rebound analyte (Fig. 4A(a)), as illustrated in the metronidazole-imprinted fluorescence sensor; an electron of metronidazole would transfer to and occupy the vacancy of graphene QDs and thus quench the sensor (Mehrzad-Samarin et al., 2017). Likewise, the orbital can come from another part of the molecule where the fluorophore exists (i.e., the receptor of target analyte) (Fig. 4A(b)) (Valeur and Leray, 2000). As demonstrated in our previous work (Wang et al., 2016a), before the sensor rebound the target analyte (2,4-dichlorophenoxyacetic acid, 2,4-D), electron transferred from the electron-rich free primary amine of receptor (3-aminopropyltriethoxysilane, APTES) to the electron-deficient aromatic ring of fluorophore (nitrobenzoxadiazole, NBD), and hence partly quenched the fluorescence of fluorophore; after analyte rebinding, the electron-donating ability of nitrogen atoms decreased and placed receptor's HOMO in a lower energy than that of fluorophore's HOMO, and thereby switched off the PET pathway, so fluorescence was enhanced (Fig. 4 A(c)).

\subsubsection{Fluorescence resonance energy transfer}

Fluorescence resonance energy transfer (FRET), first reported by Förster (1948), can occur between two moieties which are within a certain distance of $10 \mathrm{~nm}$ and have spectral overlap between the
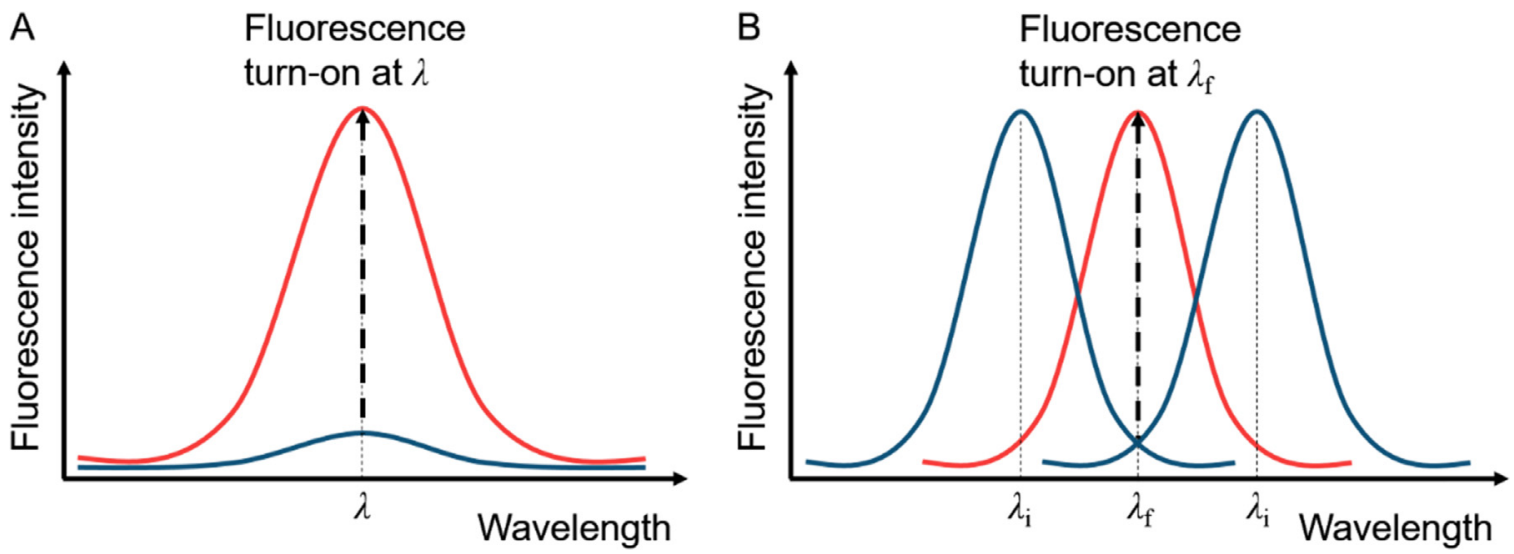

Fig. 3. "Turn-on" mode based on (A) intensity enhancement or (B) emission wavelength shift (Reproduced from Shustova et al., 2013). 
A (a)

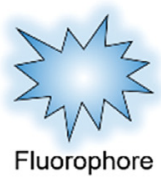

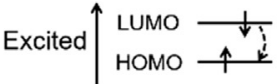

(b)

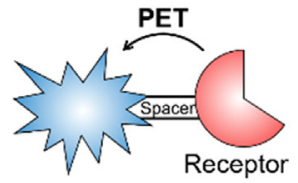

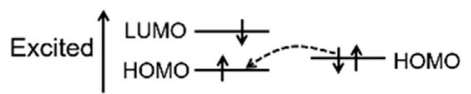

Excited

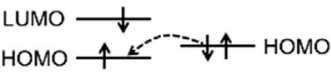

Analyte binding
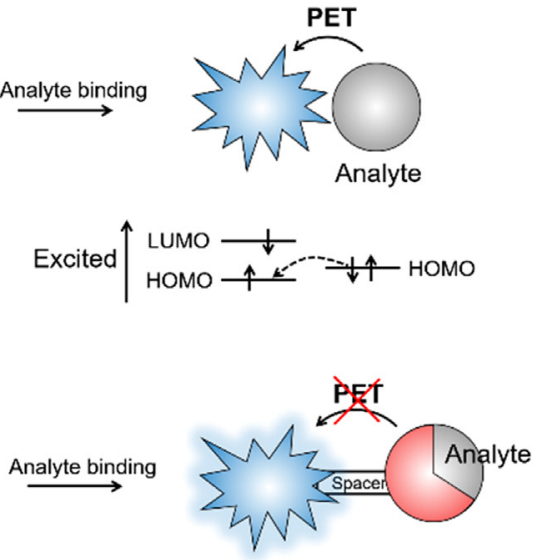

Excited $\uparrow \begin{aligned} & \text { LUMO } \frac{\downarrow}{\downarrow i} \\ & \text { HOMO } \frac{\uparrow}{4}\end{aligned}$ (c)
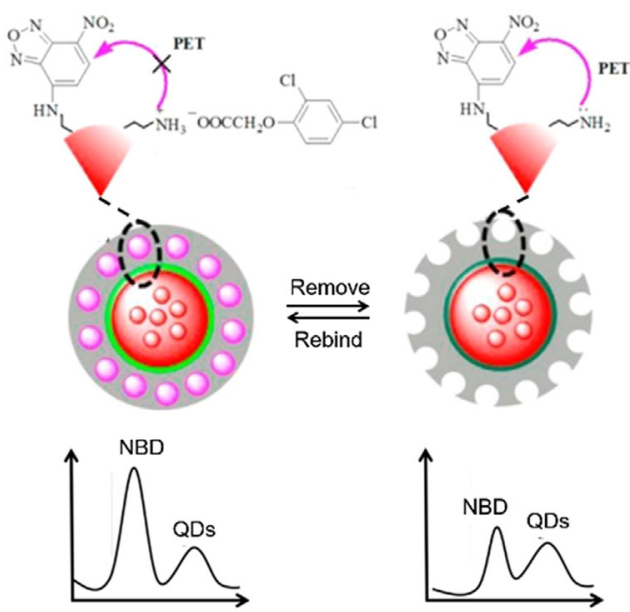

B (a)
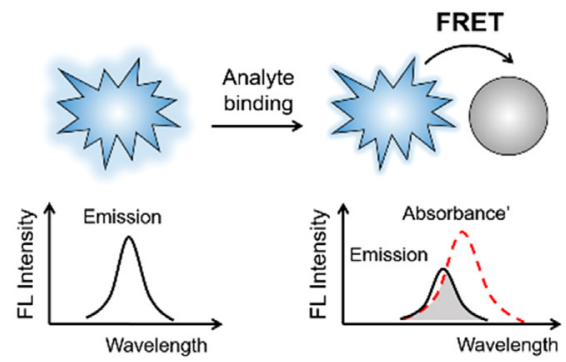

Or
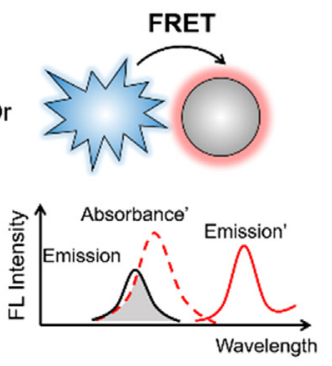

(b)

)

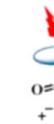

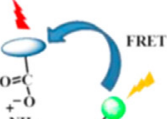
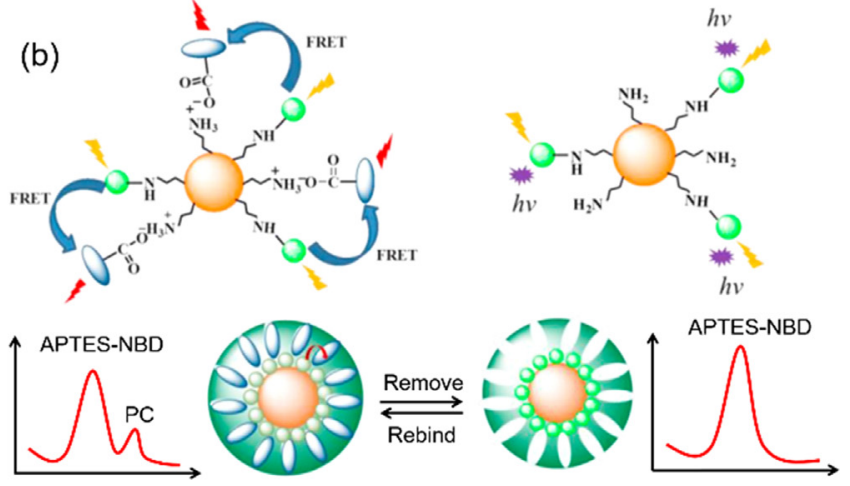

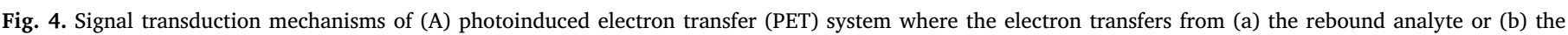

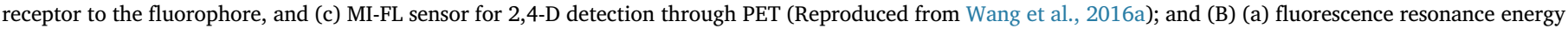

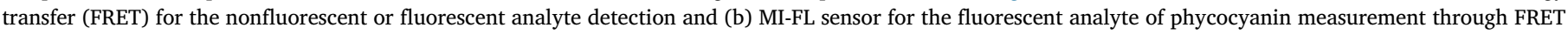
(Reproduced from Wang et al., 2016b).

emission spectrum of energy donor and the absorption spectrum of energy acceptor. Alternatively, as illustrated in Wang's work (Wang et al., 2018a), when the rebound nonfluorescent analyte (e.g., hemoglobin) acts as an energy acceptor in a MI-FL sensor, the fluorescence of the donor (i.e., the fluorophore in the sensor) would be quenched. Of course, if the analyte is intrinsically fluorescence active such as phycocyanin, not only quenching of the donor but also fluorescence appearing from the analyte would be observed in the fluorescence spectra (Fig. 4B(a)). For example, our group (Wang et al., 2016b) proposed a MI-FL sensor for phycocyanin detection. The fluorescence emission spectrum of the sensor $(500-600 \mathrm{~nm})$ overlapped the absorption spectrum of phycocyanin (500-675 nm). Hence, when they were spatially close to each other, FRET occurred from the MI-FL sensor to phycocyanin and thus quenched the fluorescence of the former (Fig. 4B(b)). Moreover, the rebound analyte can also play the role of energy donor to yield growing fluorescence of MI-FL sensors (Awino and Zhao, 2014; Li et al., 2016a).

From Förster theory (Verma et al., 2008), FRET is sensitively influenced by the distance between the donor and the acceptor. Moreover, the overlapped degree of the donor emission spectrum and acceptor absorption spectrum, and the relative orientation of the donor and acceptor dipoles both have an impact on the FRET.

Other sensing mechanisms are also reported but rarely applied in MI-FL sensors, such as intermolecular charge transfer, excimer/exciplex formation, aggregation-induced emission, $\mathrm{C}=\mathrm{N}$ isomerization, inner filter effect (Yang et al., 2017b) and so on. Hence, no further discussion is performed herein.

\section{General strategies for MI-FL sensor construction}

For targets with different native characteristics (intrinsically fluorescent active or not), the MI-FL sensors can be constructed directly by virtue of the intrinsic fluorescence of targets or constructed indirectly by relying on introduction of fluorescence-labeled analogues in competitive binding procedure and fluorescent functional monomers in imprinting procedures, as well as various fluorescence substances embedded in sensors, as shown in Fig. 5.

\subsection{Direct construction}

Some molecules are intrinsically fluorescent because of their combined aromatic groups or other rigid plane structure with several conjugated double bonds (Miller et al., 2010). These fluorescent molecules can act as templates in common MIPs preparation, and output fluorescence signals including fluorescence intensity (Carrasco et al., 2014), polarization (Ton et al., 2012) and lifetime (Urraca et al., 2014) during the rebinding procedure for quantification (Fig. 5A).

Among them, fluorescence intensity measurement seems to be easy for operation and observation. However, a tedious bound/free separation before fluorescence intensity measurement is demanded because the analyte-bound MIPs, as well as the coexisting free analyte, and analogues can emit similar fluorescence. For separation-free detection 
A
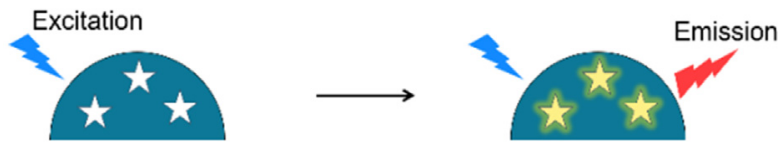

B
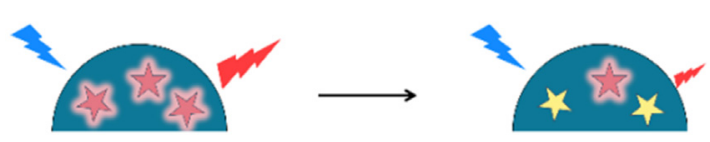

C
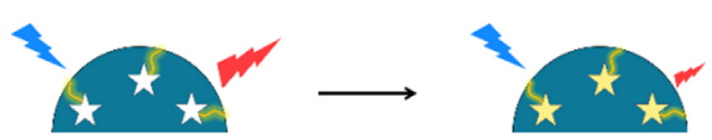

D
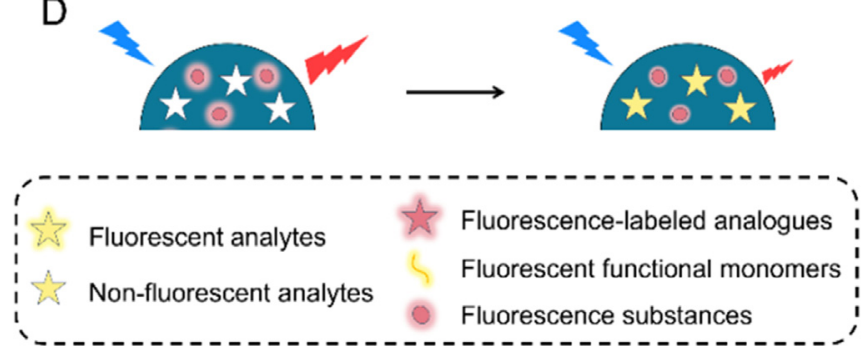

Fig. 5. General strategies for MI-FL construction of (A) direct construction and indirect construction by introducing (B) fluorescence-labeled analogues, (C) fluorescent functional monomers or (D) various fluorescence substances.

of fluorescent enrofloxacin, Ton et al. (2012) drew support from fluorescence polarization analysis: fast tumbling rate of the excited fluorescent molecules would make themselves orientate randomly and thus lower the polarization value by depolarized emission. Upon excitation by plane-polarized light, the tumbling rate of free excited enrofloxacin in solution was rapid so that the polarization value was negligible; inversely, enrofloxacin-MIP complex corresponded to lower tumbling rate but higher polarization value via polarized emission. Moreover, through fluorescence lifetime image technology with single photon timing measurements, Moreno-Bondi's group (Urraca et al., 2014) fabricated MIP patterns with submicron lateral resolution by photoinduced imprinting R123 within metallic subwavelength apertures, and the native fluorescence allowed sensitive detection of the binding events.

To sum up, depending on the native fluorescence of targets, direct construction of MI-FL sensors is more easily operational because it substantially equals to constructing traditional MIPs. However, only a fraction of analytes possesses intrinsic luminescence so that this method is of narrow applicability.

\subsection{Indirect construction}

For nonfluorescent analytes, in general, no fluorescence signal can be output after specific binding if no exotic fluorescence source is doped into MIPs. Three methods are commonly used to introduce extrinsic fluorescence as below, along with improvement in operability and applicability.

\subsubsection{Competitive binding against pre-bound fluorescent indicators}

The procedure to realize competitive binding can be described as prepare MIPs using target analytes as the templates first; saturate the imprinted cavities with fluorescent indicators (namely, fluorescencelabeled analytes or fluorescent analogues) second; and replace these fluorescent indicators by target analytes through binding cavities competition finally, with fluorescence characteristic changing depending on how much the fluorescent indicators are replaced. The elemental procedure is schematically shown in Fig. 6. Obviously, target analytes can easily enter the specific recognition sites with a remarkable competitive advantage against fluorescence-labeled analytes or fluorescent analogues (Ming et al., 2017). As early as in 1997, Piletsky et al. (1997) detected triazine based on binding cavities competition between fluorescein-labeled triazine and unlabeled triazine. After competitive adsorption, MIPs were separated by centrifugation, and the fluorescence intensity of supernatant containing released fluoresceinlabeled triazine was measured. The more triazine participated in the competition, the more fluorescein-labeled triazines were released in the supernatant, and the stronger fluorescence intensity was measured. For simplifying the MIPs separation procedure after competitive binding, our group (Ming et al., 2017) constructed MIPs on the surface of $\mathrm{Fe}_{3} \mathrm{O}_{4}$ to detect $17 \beta$-estradiol, replacing the centrifugation with separation in an additional magnetic field.

Likewise, fluorescence polarization measurement can avoid the separation step in the competitive assay, demonstrated by Murase's work (Murase et al., 2016): free cortisol (target analyte) replaced the prebound dansyl-cortisol, resulting in an increase of free dansyl-cortisol in samples to decrease the fluorescence anisotropy value. The decrease was proportional to the concentration of cortisol and thereby quantitative detection can be attained.

Actually, it is difficult to control the content of the fluorescent indicator for saturating the imprinted cavities: once the content was excessive, the excess of the unbound fluorescent indicator in the test solution would generate high background fluorescence, causing little change in fluorescence measurement after competitive adsorption and thus decreasing the sensitivity; similarly, a deficiency of the fluorescent indicator cannot fully saturate the imprinted cavities, narrowing the detection range. Moreover, the incubation of fluorescence-labeled analytes and further competitive binding with the target analytes is time-consuming. Construction of an appropriate fluorescent indicator with a similar structure to the target is also not easy.

\subsubsection{Employment of fluorescent functional monomers}

Employing fluorescent functional monomers to replace the traditional functional monomers or act as co-monomers is promising for preparing MI-FL sensors for nonfluorescent analytes determination, through monitoring the fluorescence change after interaction between fluorescent functional monomers and analytes. A fluorescent functional monomer always possesses three moieties: a polymerizable moiety as a reactive linker to form MIP matrix together with cross-linker, a recognition moiety to recognize targets under a specific force such as hydrogen bonds, and a fluorophore for transducing binding events into fluorescence signals. As summarized in Fig. 7, fluorescent functional monomers can be synthesized by two typical approaches: (i) derivatization of a fluorophore with a polymerizable moiety and a recognition moiety (Fig. 7A-E); and (ii) grafting a traditional functional monomer with a fluorophore (Fig. 7F-H).

By deriving a fluorophore of acenaphthene through four steps (Fig. 7A), Rouhani and Nahavandifard (2014) synthesized the fluorescent functional monomer N-allyl-4-ethylenediamine-1,8-naphthalimide to participate in the MI-FL sensor construction for caffeine detection. Obviously, the derived $\mathrm{N}$-allyl group could be polymerized with cross-linker of ethylene glycol dimethacrylate to form a rigid MIP matrix, the derived 2-aminoethylene at C4-position of naphthalimide ring could recognize caffeine through hydrogen bonds, and the inherent naphthalimide ring was responsible for generating fluorescence. The PET mechanism explained the fluorescence quenching rather than fluorescence enhancement upon analyte bonding: before binding, an electron transferred from receptor to the excited naphthalimide fluorophore made fluorescence quenching; after binding, the HOMO of the receptor increased because of the formation of the caffeine-receptor complex, leading to more feasible electron transfer to strengthen fluorescence quenching. Other related fluorophore-derived fluorescent functional monomers have also been reported by Lu et al. (2018); Qiu et al. (2017); Chen et al. (2014) and Wagner et al. (2013), using the 


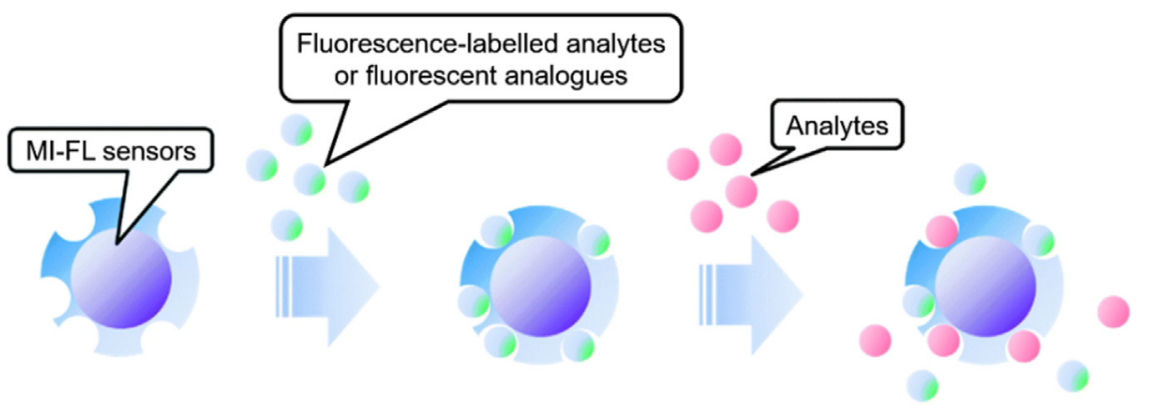

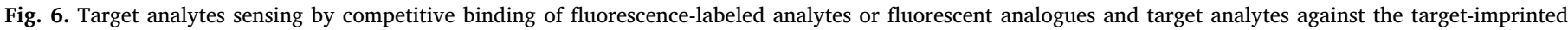
cavities in MI-FL sensors. (Reproduced from Murase et al., 2016).

basic derivatization procedures shown in Fig. 7B-E, respectively.

Through modifying a traditional functional monomer of 2-hydroxyethyl methacrylate (HEMA) with a fluorophore of anthracene-9-carboxylic acid (Fig. 7F), Niu et al. (2015) prepared a fluorescent functional monomer of (2-hydroxyethyl anthrancene-9-carboxylate) methacrylate (AnHEMA), and then imprinted it in a hydrophilic MI-FL sensor for tetracycline detection. Charge transfer from the sensor to the rebound tetracycline led to fluorescence quenching. Impressively, the sensor was surface-grafted with hydrophilic poly(2-hydroxyethyl methacrylate) brushes so that it was greatly improved in surface hydrophilicity and extremely suitable to detect tetracycline in real, undiluted biological samples (including bovine and pig serum) for their macromolecule filterability. Similarly, Wan et al. (2013) and Wu et al. (2015a) grafted the traditional methyl methacrylate-based functional monomers with suitable fluorophores, as shown in Fig. $7 \mathrm{G}$ and $\mathrm{H}$, respectively.

However, complicated synthesis procedures of fluorophore derivatization and endowing nonfluorescent functional monomers with fluorophores and different synthesis methods of specific fluorescent functional monomers for various templates, as well as the photobleaching problem of the organic fluorophore, all limit the application of the fluorescent functional monomer in MI-FL sensor.

\subsubsection{Embedding of fluorescence substances}

Apart from the complex construction of competitive binding and synthesis of fluorescent functional monomers, another frequently used and simple procedure to develop MI-FL sensors is to embed fluorescence substances, i.e., fluorescent dyes, quantum dots (QDs), metal nanoclusters (NCs) and lanthanide chelates. The features of these different fluorescent materials are summarized in Table 1 , to give a comparison of their own advantages and limitations.

3.2.3.1. Fluorescent dyes. Fluorescent dyes always contain one or more fluorophores. Before the rise of new fluorescence materials, fluorescence dyes including fluorescein isothiocyanate (FITC) (Sunayama et al., 2010), porphyrins (Johnson-White et al., 2007), nitrobenzoxadiazole (NBD) (Shinde et al., 2015) and dansyl (IvanovaMitseva et al., 2012) had been widely utilized in MI-FL sensors to provide fluorescence signals upon analytes binding for their wide varieties, rich colors and easy accessibility. For example, Li et al. (2015b) electropolymerized a neutral red-doped fenaminosulfimprinted film on the silver-nanofilm-modified electrodes, where neutral red and fenaminosulf formed ion pair to diminish the fluorescence. The silver nanofilm effectively amplified the fluorescence quenching by enhancing the redox activity of neutral red, facilitating the ultrasensitive fenaminosulf measurement down to $1.6 \times 10^{-11} \mathrm{~mol} \cdot \mathrm{L}^{-1}$. Sreenivasan's group (Manju et al., 2010) constructed a fluorescein o-acrylate embedded film-like MI-FL sensor for glucose estimating in tear fluid, where one functional monomer of trans-2-chloromethyl vinyl boronic acids took charge of glucose

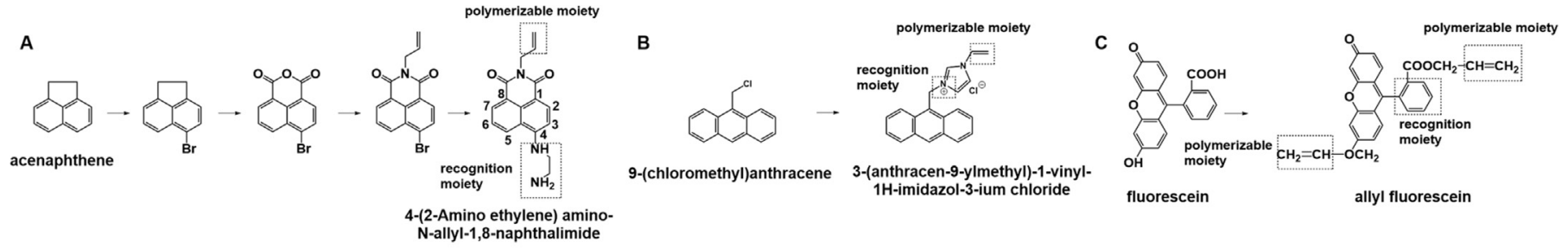

D

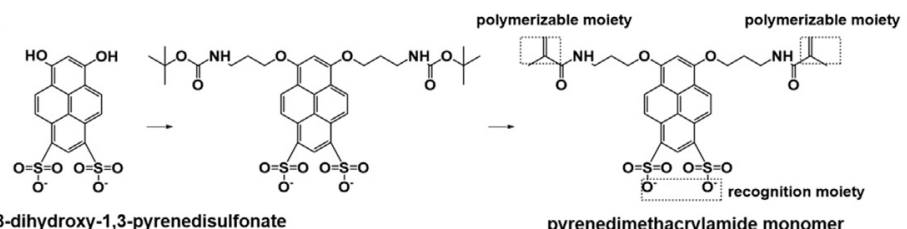

E

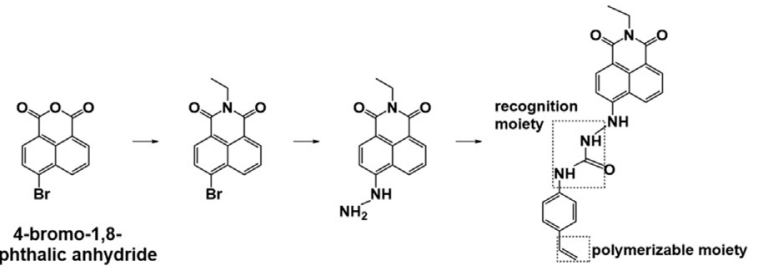

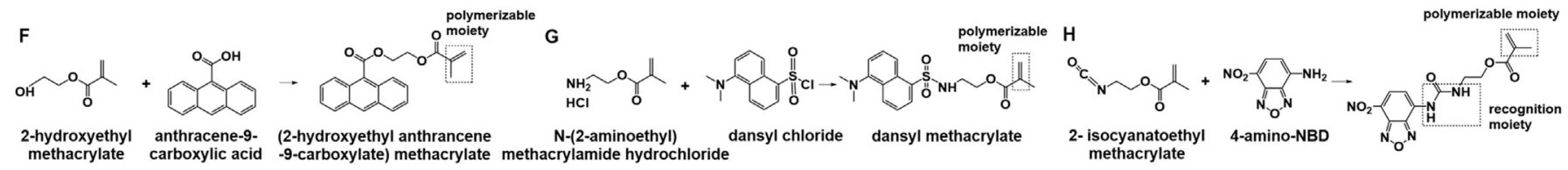

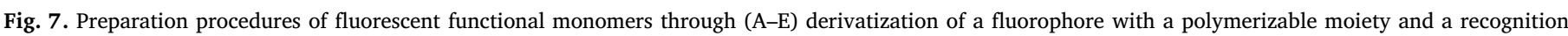

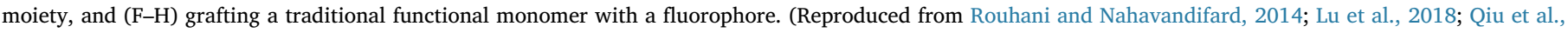
2017; Chen et al., 2014; Wagner et al., 2013; Niu et al., 2015; Wan et al., 2013; Wu et al., 2015a, respectively). 
Table 1

Advantages and limitations of various fluorescent materials embedded in MI-FL sensors.

\begin{tabular}{|c|c|c|}
\hline Material type & Advantages & Limitations \\
\hline Organic fluorescence dyes & $\begin{array}{l}\text { Wide varieties, rich colors, easy access, small size, good biocompatibility and relatively } \\
\text { high fluorescent intensity }\end{array}$ & $\begin{array}{l}\text { Narrow excitation peak, broad and tailing emission } \\
\text { peak, poor stability, easy photobleaching }\end{array}$ \\
\hline Quantum dots (QDs) & $\begin{array}{l}\text { (i) Semiconductor QDs: good chemical stability, wide excitation peak, narrow and } \\
\text { symmetrical emission peak, strong luminescence, large Stokes shift and color tenability; } \\
\text { (ii) Carbon-based QDs: low toxicity, biocompatibility, good chemical stability and } \\
\text { comparable photoluminescence properties against QDs }\end{array}$ & $\begin{array}{l}\text { Heavy-ion containing QDs: toxicity and fluorescence } \\
\text { intermittence }\end{array}$ \\
\hline Metal nanoclusters & $\begin{array}{l}\text { Low toxicity, ease preparation, excellent biocompatibility, and compared with QDs, } \\
\text { exhibit stronger and more durable fluorescence signals attributed to better resistance to } \\
\text { photobleaching and blinking }\end{array}$ & Noble metals nanoclusters are expensive. \\
\hline Upconversion nanoparticles & $\begin{array}{l}\text { Deeper light penetration, lack of autofluorescence (from biological samples), long lifetimes } \\
\text { and other excellent optical property of metal nanoclusters }\end{array}$ & - \\
\hline
\end{tabular}

recognition through the affinity of boronic acid moieties toward diols. Before glucose rebinding, fluorescein o-acrylate was surrounded by boronic acids moieties through hydrogen bonds; after interaction between glucose and boronic acids, the conformation of fluorophorecontaining polymer chains changed and thus the MI-FL sensor was quenched. A more hydrophilic environment created by the adsorption of water molecules also quenched the hydrophobic fluorophore.

However, some insurmountable problems of traditional fluorescence dyes such as high background signal, wide excitation spectra, easy and rapid photobleaching and so on make them unsuitable in the MI-FL sensor for accurate, long-term and sustainable use (Schrum et al., 2000). Hence, new fluorescence materials such as QDs, metal NCs and lanthanide chelates have emerged.

3.2.3.2. Quantum dots. Inorganic semiconductor QDs, discovered in the early 1980s (Brus, 1983; Efros and Efros, 1982), are composed of hundreds to many thousands of atoms from IIB-VIA or IIIA-VA, with confined sized on nanoscale in three dimensions to show a remarkable quantum confinement effect (Zhou et al., 2015a). Compared with organic fluorescent dye, QDs are more photostable and can exhibit strong luminescence, wide excitation but sharp emission profile, which are size-dependent (Silvi and Credi, 2015). The most well-known QDs embedded in MI-FL sensors are cadmium (Cd) (Huy et al., 2014; Liu et al., 2014b) and zinc (Zn) based (Chantada-Vázquez et al., 2016; Chen et al., 2017a; Li et al., 2017a; Zhang et al., 2018), and as we know, QDs synthesized using hydrophobic ligands such as trioctyl phosphine oxide (TOPO) can endow QDs with excellent quantum yield and chemical stability, but limit their hydrophilic applications. Using a reverse microemulsion polymerization, Liu et al. (2014b) embedded the hydrophobic TOPO-modified CdSe QDs into the MI-FL sensor using cyclohexane as a continuous phase and Triton X-100 as a surfactant, obtaining both high quantum yield and hydrophilic applicability for ractopamine detection. For further enhancing the convenience of application in aqueous environments and possessing a high fluorescence quantum yield, QDs are synthesized in the aqueous phase with the modification of various capping reagents. Taking CdTe QDs as an example, Huy et al. (2014) capped them with mercaptoacetic acid to increase surface tension and hinder growth and further anchored clenbuterol or melamine imprinted layers on the surface of them via sol-gel polymerization. Instead of going back to the valence band, the charges at the conduction band of CdTe QDs transferred to the rebound clenbuterol or melamine, so the fluorescence linearly quenched with analyte concentration increase. Other related hydrophilic ligand-modified QDs are also presented (Chao et al., 2014; Qiu et al., 2015; Xu et al., 2013; Yang et al., 2016; Yu et al., 2017).

For the intrinsic toxicity of the traditional heavy-ion-based QDs (Yong et al., 2013), newly eco-friendly carbon-based QDs, namely, graphene quantum dots (GQDs) and carbon quantum dots (CQDs), are preferred to be utilized in the MI-FL sensors.

GQDs, a zero-dimensional graphitic nanomaterial found in 2004
(Novoselov et al., 2004), is one type of carbon-based QDs with lateral dimensions less than $100 \mathrm{~nm}$ to possess nanosize $\mathrm{sp}^{2}$ domains (Lin et al., 2014). Currently, two possible mechanisms are used to explain its photoluminescence mechanism: band-gap transition and electron-hole recombination relevant to the presence of defects (Yoo et al., 2015). GQDs are always synthesized by thermo-oxidatively cutting of graphene oxides (GOs) or other carbon precursors. Through pyrolyzing citric acid, for instance, Mehrzad-Samarin et al. (2017) synthesized the GQDs and further modified them with APTES for easily anchoring silica MIPs. The PET between GQDs and the rebinding metronidazole resulted in linear fluorescence quenching. Similarly, Zhou et al. (2014) hydrothermally deoxidized APTES-GO and embedded the obtained GQDs into the MI-FL sensor via a typical sol-gel polymerization. Resonance energy transfer from GQDs to the rebound 4-nitrophenol resulted in fluorescence quenching of GQDs.

CQDs, found in the same year of 2004 (Xu et al., 2004) as GQDs, are another type of carbon-based QDs with size within $10 \mathrm{~nm}$. CQDs could be easily prepared by breaking larger carbon structures such as graphite and carbon nanotubes through arc discharge, laser ablation and electrochemical oxidation ("top-down" route), or by combustion/thermal treatments of molecular precursors such as citrate, carbohydrates and polymer-silica nanocomposites ("bottom-up" route) (Jalili and Amjadi, 2018; Lim et al., 2015; Wang and Hu, 2014). Two possible fluorescence emission mechanisms of CQDs have been reviewed: band-gap transitions caused by conjugated pi-domains, and more intricate origins associated with surface defects of CQDs (Chen et al., 2017b; Lim et al., 2015). Feng et al. (2015) synthesized vinyl group-modified CQDs using citric acid as a carbon source and further proposed the CQDs-containing sensor via epitope imprinting, where the co-monomers of methacrylic acid and 4-Vinyl pyridine were screened out by computer simulation. The doped CQDs acted as antennae for recognition signal amplification and optical readout on the a-amanitin binding event. Furthermore, Li et al. (2016b) prepared a MI-FL sensor embedded with the no further post-modification CQDs: the carboxyl groups of pyrolysis citric acid reacted with amine groups of $\mathrm{N}$-( $\beta$-aminoethyl)- $\gamma$-aminopropyl methyldimethoxysilane to synthesize the organosilane-functionalized CQDs, and then, the CQDs were condensed with cross-linker to form a $\mathrm{Si}-\mathrm{O}-\mathrm{Si}$ framework in a one-pot non-hydrology sol-gel reaction, imprinting the 1,8-dihydroxyanthraquinone (an alternative template to sterigmatocystin) simultaneously. The regular fluorescence quenching of the encapsulated CQDs may result from the excited electron transferring from CQDs@MIP to sterigmatocystin.

3.2.3.3. Metal nanoclusters. NCs, a new type of fluorescent nanomaterial, usually comprise several to tens of atoms with ultrasmall size within $5 \mathrm{~nm}$ so that they are considered the missing link between metal atoms and nanoparticles. Resulting from the ultra-small size comparable to the Fermi wavelength of electrons, the discrete energy levels of NCs afford themselves with the size-dependent fluorescence (Zheng et al., 2007). In addition to the comparable fluorescent property to QDs (Li et al., 2016c), the NCs are of low 
cytotoxicity, biocompatibility and environmental friendliness. The most widely used method to prepare NCs, particularly the gold nanoclusters (AuNCs) and silver nanoclusters (AgNCs), is the reduction of metal ions in aqueous solution with suitable agents (e.g., L-Glutathione) to stabilize NCs and enhance fluorescence (Shang et al., 2011).

Compared with other NCs, AuNCs exhibit the stronger and more durable fluorescence signals attributed to lower surface activation energy and harder aggregation in aqueous solution. For example, in an AuNCs-supported bisphenol A (BPA) imprinted layer, strong charge transferred from the electron-rich aromatic ring of BPA to the electron deficient amino group of AuNCs and generated a Meisenheimer complex to accept the photoluminescent energy of AuNCs, resulting in fluorescence quenching of AuNCs (Wu et al., 2015b). Similarly, through surface imprinting on AgNCs cores, the rebound BPA also quenched AgNCs through excited charge transfer from the conductive bands to BPA (Deng et al., 2016). However, the high cost of preparation and application of noble metal NCs cannot be neglected. The much less expensive copper nanoclusters (CuNCs) might be a good alternative to replace them, but their easier oxidation in air than AuNCs and AgNCs would largely quench their fluorescence and induce aggregation, limiting the fabrication and further functionalization of ultra-small CuNCs and application in MI-FL sensors (Li et al., 2016c; Liu et al., 2015).

3.2.3.4. Lanthanide chelates and upconversion nanoparticles. Lanthanide elements, i.e., rare earth elements, possess notable luminescent properties due to their unique unsaturated $4 f$ orbitals, where electron transition would happen in different energy levels to produce fluorescence. The $f-f$ transitions are usually Laporte-forbidden but allowed in lanthanide elements, and therefore, (i) the transition probability is low, so the lifetime is long $\left(10^{-6}-10^{-2} \mathrm{~s}\right)$; (ii) excitation energy is hard to be absorbed so that the luminescence efficiency is low, and hence, its alternative is to develop lanthanide chelates to enhance fluorescence efficiency. Among various lanthanide chelates, europium (III) $\left(\mathrm{Eu}^{3+}\right)$ chelates are preferred to be incorporated into MI-FL sensors for wide excitation band, narrow emission band and long fluorescence lifetimes (Liu et al., 2013, 2014a; Zheng et al., 2015). For instance, Liu et al. (2013) designed the mesoporous silica spheres covalently modified with red luminescent $\mathrm{Eu}^{3+}$ complex $\left(\mathrm{mSiO}_{2} \text {-Eu(TTA) }\right)_{3} \mathrm{Bpc}$ ), and then the $\mathrm{mSiO}_{2}$-Eu(TTA) $)_{3} \mathrm{Bpc}$ acted as stabilizing particles during the MI-FL sensor preparation by Pickering emulsion polymerization as well as provided decreasing fluorescence intensity upon $\lambda$-cyhalothrin recognition.

In addition, lanthanide-doped upconversion nanoparticles (UCNPs) is newly utilized in MI-FL sensor, which could convert low-energy light (near-infrared or infrared light) into high-energy light (UV-visible light) through multiple photon absorptions, energy transfers or photon avalanches (Auzel, 2004). Compared with UV-visible light, the nearinfrared or infrared light can obtain deeper penetration into samples without photodamage, and avoid autofluorescence from a coexisting substance. Additionally, UCNPs could emit narrow-band and long-lifetime fluorescence with low toxicity and photobleaching (Tang et al., 2015). In particular, efficient upconversion process could be realized only by doped trivalent lanthanide ions (e.g., $\mathrm{Eu}^{3+}$ ) owing to their long lifetime at a metastable level to allow absorbing the next electron before emission. According to the literatures, the reported UCNPs-embedded MI-FL sensors are mainly constructed by Li's and Wang's groups (Guo et al., 2015, 2016a, 2016b; Qian et al., 2013; Tang et al., 2015). Initially, two MI-FL sensors (Guo et al., 2015; Tang et al., 2015) were both proposed by surface imprinting on UCNPs of $\mathrm{YF}_{3}: \mathrm{Yb}^{3+}$ and $\mathrm{Er}^{3+}$ by free radical polymerization and sol-gel polymerization, respectively, where the $\mathrm{NaYF}_{4}$ has become the most efficient host material for UCNPs currently (Boyer et al., 2010). Afterward, Wang's group (Guo et al., 2016a) prepared a thermosensitive MI-FL sensor with UCNPs/HKUST-1 as the core, where HKUST-1 was added as a representative metal-organic framework (MOF) and prepared by the reaction of copper nitrate with benzene tricarboxylate on the surface of UCNPs through a solvothermal method. The high specific surface area and porosity of HKUST-1, excellent fluorescence of UCNPs and super selectivity of MIPs have endowed the MI-FL sensor with satisfactory sensing properties to bovine serum albumin. At the same time, Wang's group (Guo et al., 2016b) developed a dual-responsive (pH and temperature) MI-FL sensor with grapheme oxide (GO, with ultrahigh specific surface area) as support material and UCNPs as fluorescence signal reporter for quantitative determination of horse radish peroxidase. And recently, Li's group (Liu et al., 2017) developed a double recognition aptamerMIP/UCNPs sensor on UCNPs to detect enrofloxacin, in which aptamerlinked UCNPs were first obtained by immobilizing enrofloxacin aptamers through biotin-avidin affinity reaction before the surface imprinting. After eluting, imprinted cavities could dually recognize enrofloxacin owing to the enrofloxacin aptamer and MIPs, and the dual recognition was confirmed by comparison of aptamer-MIP/UCNPs against MIP/UCNPs.

\subsection{Other methods}

Differing from the above methods, Subrahmanyam et al. (2000) have prepared a MI-FL sensor by polymerizing ally mercaptan, $o$ phthalic dialdehyde and methylated creatine (dummy template). Herein, the interaction between ally mercaptan and $o$-phthalic dialdehyde formed the polymerizable hemithioacetal, which could irreversibly react with primary amine-containing creatine to produce fluorescent isoindole, generating a detection signal upon specific analyte rebinding. The reason to use methylated creatine as a template is that methylated creatine could form a reversible complex with hemithioacetal, leading to the easy removing of the template and the forming of imprinted cavities.

To sum up, direct or indirect construction of MI-FL sensors are promising for selectively and sensitively detecting fluorescent or nonfluorescent analytes. However, the sensing performance involving responsive time, sensitivity, specificity and detection range all remain to be improved owing to some limitations: (i) large mass-transfer resistance and inaccessibility recognition sites in solid spheres or irregular bulk particles; (ii) intrinsic high background fluorescence; (iii) random location of fluorescent indicators outside the imprinted cavities; and (iv) interference from various analyte-independent factors.

\section{Novel strategies for MI-FL sensor construction}

For MI-FL sensors, renovation in both molecular imprinting and fluorescence detection are promising for enhancing their sensing performance. Hierarchical architecture construction such as core-shell, hollow, and mesoporous structure, post-imprinting modifications (PIM), and ratiometric fluorescence are the three major novel construction strategies to improve the sensing properties of MI-FL sensors.

\subsection{Hierarchical architecture construction}

In traditional solid-sphere or irregular bulk-particle MI-FL sensors, recognition sites are deeply embedded in the highly cross-linked matrix, generating inaccessible imprinted cavities and big mass transfer resistance, as schematically shown in Fig. 8A. It is promising to make recognition cavities accessible and reduce mass transfer resistance by upgrading the morphology of sensors, such as core-shell (Fig. 8B), hollow (Fig. 8C) and core-shell-based mesoporous structures (Fig. 8D).

\subsubsection{Core-shell and hollow structure}

Core-shell architecture construction means anchoring the fluorescent MIPs layer on the surface of a proper support material, leaving imprinted cavities suited at the surface or in the proximity of the surface to provide sites accessibility and lower mass transfer resistance, as illustrated in Fig. 8B. The commonly reported support materials are silica (Ivanova-Mitseva et al., 2012; Li et al., 2013; Shinde et al., 2015; 

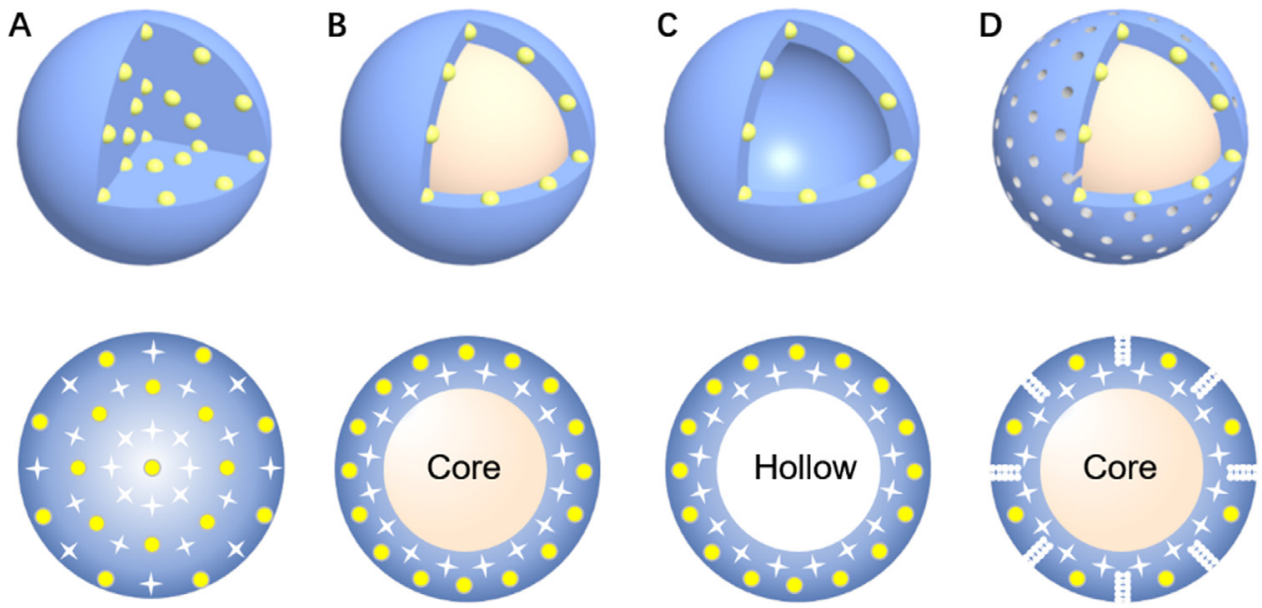

Fig. 8. MI-FL sensors with various morphologies of (A) solid sphere or irregular bulk particle, (B) core-shell, (C) hollow, (D) core-shell-based mesoporous structures (upper: space illustration; bellow: planar illustration).

A
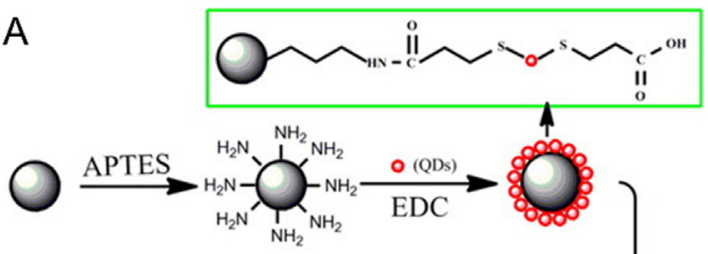<smiles>CC[Si](C=O)(C=O)CCCN</smiles>

functional monomer

Light excitation

Light emission

X Suppression

B

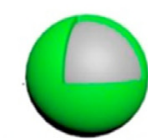
$\underset{\text { imprinting }}{\stackrel{\text { Molecular }}{\longrightarrow}}$ $\mathrm{SiO}_{2}$-APTES-FITC $\quad \mathrm{SiO}_{2} @ \mathrm{MIPs}$

- Target molecule (LC)

$\Delta \approx$ Interfering substance

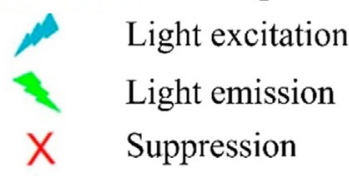

0 template
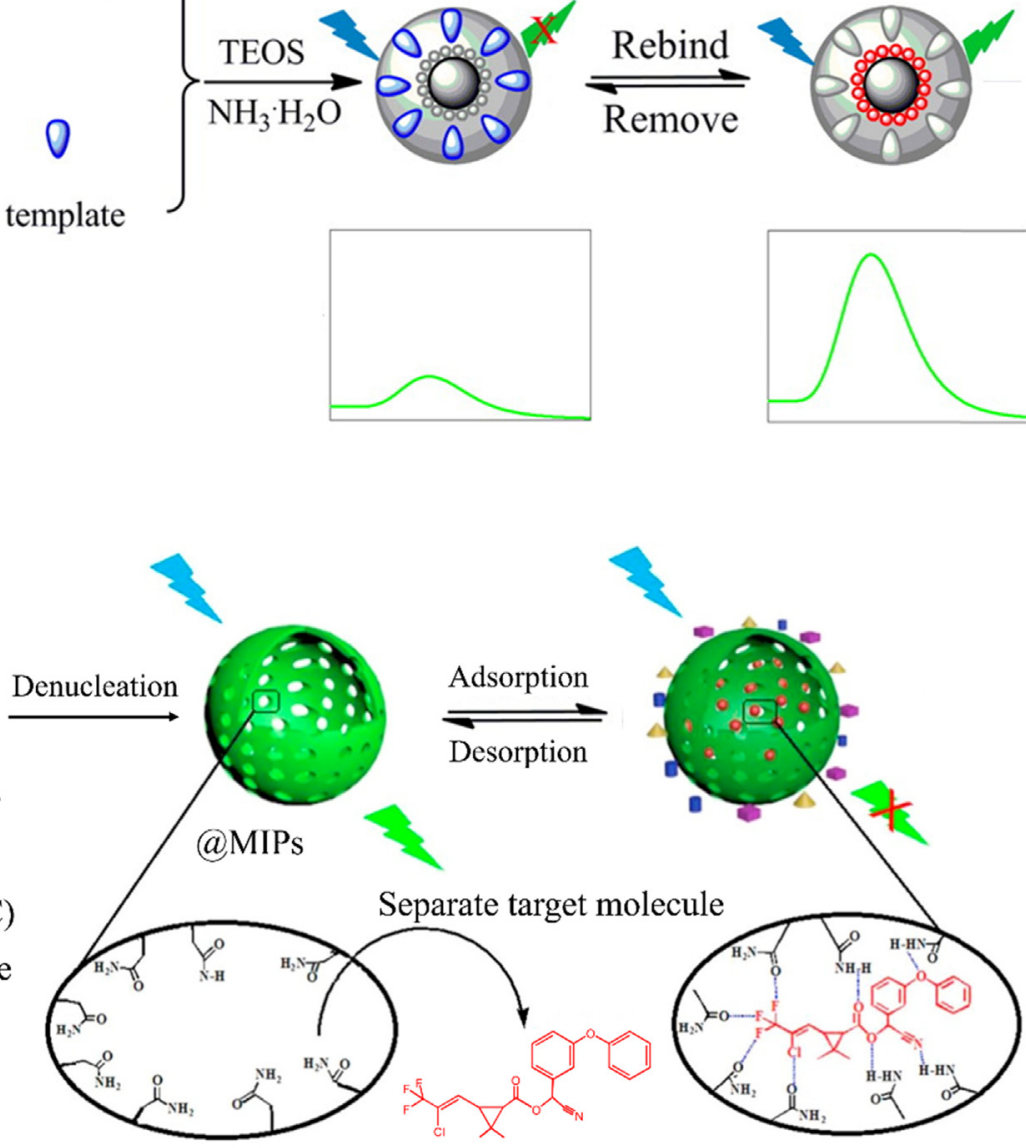

Fig. 9. Preparation procedures of the MI-FL sensor with (A) core-shell and (B) hollow structure, respectively, and their corresponding fluorescence quenching upon specific targets recognition. (Reproduced from Li et al., 2013; Wang et al., 2016c, respectively). 
Wei et al., 2018) and $\mathrm{Fe}_{3} \mathrm{O}_{4}$ nanoparticles (Li et al., 2011b; Ming et al., 2017; Zor et al., 2015). Among them, silica nanoparticles (Si-NPs) are widely used for their easy-control synthesis, high chemical/mechanical stability, biocompatibility and numerous hydroxyl groups on their surface, which can be employed as grafting sites (Li et al., 2013, 2012). Li et al. (2013) proposed the core-shell composite sensor by assembling carboxyl-terminated CdTe QDs on the surface of amino-functionalized $\mathrm{Si}$-NPs, and then encapsulating MIPs layer on the surface of SiNP@CdTe QDs, as depicted in Fig. 9A. The ultrathin layer of approximately $10 \mathrm{~nm}$ provided accessible sites and fast mass transfer, as well as well-defined material shape for specific bovine hemoglobin recognition with a linear range from 0.02 to $2.1 \mu \mathrm{mol} \mathrm{L}^{-1}$ and limit of detection (LOD) down to $9.4 \mathrm{nmol} \mathrm{L}^{-1}$. Polyindole (PIn) also uniquely acted as a core to fabricate the PIn-NP@GQDs@MIPs (Zhou et al., 2015b), where the amine groups on the PIn backbone enhanced GQDs' fluorescence intensity because of surface passivation (Wang et al., 2015). In addition, nonspherical cores such as the MOFs (Liu et al., 2018), hexagonalprism UCNPs (Tang et al., 2015, 2018), nanorod (Lee et al., 2013) and nanowire (Wei et al., 2015) can also support MIPs layer.

Furthermore, a MI-FL sensor with hollow structure (Fig. 8C) can be fabricated by etching the core, which is suspected to provide larger specific surface area apart from more accessible recognition sites and faster mass transfer. To illustrate that, a hollow MI-FL sensor was proposed by etching the silica core with hydrofluoric acid, leaving the MIPs shell to show fluorescence quenching with $\lambda$-cyhalothrin down to $10.26 \mathrm{nmol} \mathrm{L}^{-1}$, as schematically shown in Fig. 9B. The faster mass transfer was proved by fluorescent kinetic detection that hollow-MIPs and non-hollow ones could reach an equivalent fluorescence quenching at 8 and $12 \mathrm{~min}$, respectively (Wang et al., 2016c).

\subsubsection{Mesoporous structure}

Mesoporous MI-FL sensors, possessing interconnected worm-like or hexagonal-tunnel channels, facilitate the infiltration of target molecule into the imprinted sites as well. Cetyltrimethylammonium bromide (CTAB) is the common template to construct a mesoporous structure. For example, by the condensation of tetraethoxysilane in the presence of CTAB, amino-CQDs and trinitrophenol (TNP, dummy template), a mesoporous structured MI-FL sensor was proposed to quickly detect TNT within $4 \mathrm{~min}$, where amino-CQDs acted as both functional monomer and fluorescent reporter to guarantee each imprinted cavity surrounded by fluorescent reporters (Fig. 10A). The mesoporous structured sensor was endowed with remarkably lower LOD $\left(17 \mathrm{nmol} \mathrm{L}^{-}\right.$ $\left.{ }^{1}\right)$ compared with the non-mesoporous structured one $\left(190 \mathrm{nmol} \mathrm{L}^{-1}\right)$ (Xu and Lu, 2016).

By combining core-shell architecture with mesoporous structure (Fig. 8D), Zhang et al. (2015a) developed Si-NP@CdTe QD@mes-MIPs to detect physocyanin down to $5.9 \mathrm{nmol} \mathrm{L}^{-1}$, through linking many carboxylated-QDs on the surface of amino-functionalized Si-NPs and following depositing mesoporous structured imprinting shell (approximately $5 \mathrm{~nm}$ ) on the surface (Fig. 10B). In addition, compared with the non-mesoporous structured one, the mes-MIPs shortened the response time from $10 \mathrm{~min}$ to $4-8 \mathrm{~min}$. Additional work based on the core-shell and mesoporous structure has also been conducted for 2,4-D detection (Jia et al., 2017).

\subsection{Post-imprinting modification}

Fluorescent reporters are always mixed in the imprinting system to propose MI-FL sensors, and resultantly, they are immobilized randomly, and a number of them are outside the imprinted cavities to cause high fluorescence background. Moreover, some analytes bound not by imprinted cavities can also be transduced into fluorescence signals to generate nonspecific recognition. Post-imprinting modification (PIM), defined as site-specific chemical modifications to introduce additional fluorescent function within the pre-constructed molecular imprinting cavities (Takeuchi et al., 2015), is promising for reducing background fluorescence and weakening the nonspecific sensing since these fluorescent reporters respond only to the target molecules bound within imprinted cavities. The theory has been demonstrated by comparison with the surface plasmon resonance (SPR) measurement both in imprinting factor (defined as the ratio of binding constants between imprinted and non-imprinted polymers) and selectivity (Sunayama et al., 2010, 2014), in which for the SPR method, analyte detection is a summation of specific and nonspecific analyte binding.

Takeuchi's and Wang's groups have made remarkable contributions to the development of PIMs in MI-FL sensors by employing directly modifiable functional monomers or cleavable functional monomers. Initially, Takeuchi et al. (Sunayama et al., 2010) directly modified the secondary amine group of the functional monomer (4-[2-(N-methacrylamido)-ethylaminomethyl]benzoic acid, MABA) with an amine-reactive fluorophore of FITC, to introduce fluorescent reporter for lysozyme detection (Fig. 11A(a)). Afterward, additionally based on MABA to detect lysozyme, these researchers further reduced the background fluorescence and improved the specificity by a two-step PIMs (Sunayama et al., 2014). Briefly, after lysozyme at an ultralow concentration occupied the high-affinity binding cavities, amine-reactive $p$ isothiocyanatophenyl $\alpha$-D-mannopyranoside (MITC) capped both lowaffinity binding cavities and erroneous MABA residues outside of the binding cavities, defined as the first PIM. Next, lysozyme was removed to regenerate the high-affinity binding cavities, and the amine-reactive FITC was then introduced into these high-affinity binding cavities as the second PIM (Fig. 11A(b)). The first PIM prevented the inaccurate label of FITC to undesirable position and consequently enhanced the sensitivity, demonstrated by two experimental facts: (i) the MITC-capped sensor showed $37 \%$ lower background fluorescence intensity than the uncapped sensor; (ii) the MITC-capped sensor exhibited higher fluorescence intensity change than the uncapped sensor with the increasing concentration of lysozyme injected.

However, it is difficult to maintain stable interaction between the amine-containing functional monomers and basic proteins (lysozyme) during the imprinting procedure on account of the electrostatic repulsion; thus, for higher affinity binding, amine-containing functional monomers can be replaced by the cleavable functional monomer of ( $\{$ [2-(2-methacrylamido)ethyldithio]-ethylcarbamoyl $\}$ methoxy)acetic acid (MDTA) (Sunayama and Takeuchi, 2014). After removal of lysozyme, the disulfide linkages were cleaved to expose thiol residues, followed with a disulfide exchange to attach aminoethyl groups for labeling amine-reactive FITC, yielding specific fluorescence enhancement with increasing lysozyme (Fig. 11B(a)). Excitingly, owing to the easy-achieved disulfide exchange reactions, the disulfide linkage is similar to a screwdriver that can replace different screw heads to transform or exchange the original functionality into different functions, not merely the aminoethyl group. Alternatively, more simply, the exposed thiol residues can be modified with a thiol-reactive fluorescent reporter, e.g., 4-(N,N-dimethylaminosulfonyl)-7-fluoro-2,1,3-benzoxadiazole (DBD-F) (Fig. 11B(b)) (Suga et al., 2013). Unlike modifying fluorescent materials, Wang's group (Zhao et al., 2017) labeled the nonfluorescent 4-vinyl phenylboronic acid to the free thiol group for nonfluorescent horseradish peroxidase (HRP) detection (Fig. 11B(c)). Upon HRP binding, 4-vinyl phenylboronic acid could not only form a five-membered ring with HRP but also improve the fluorescence intensity owing to its conjugated large pi bonds. Moreover, FRET from the post-MIPs to HRP resulted in linear fluorescence quenching with HRP increase.

Moreover, more complex functionalities can be realized by multistep PIMs. Plural fluorescent sensing, for instance, was achieved by introducing two fluorescent dyes of DBD-F and FSA (4-formylsalicylic acid) (Sunayama et al., 2016). After the MIPs construction, the exposed aniline residues were first protected by tert-butoxycarbonyl, and then amino groups were introduced to the cysteine prosthetic groups for the first fluorescent dye DBD-F modification. After deprotection of the aniline residue, FSA was conjugated via Schiff base formation as the second fluorescent dye modification (Fig. 11C). The fluorescence of FSA 

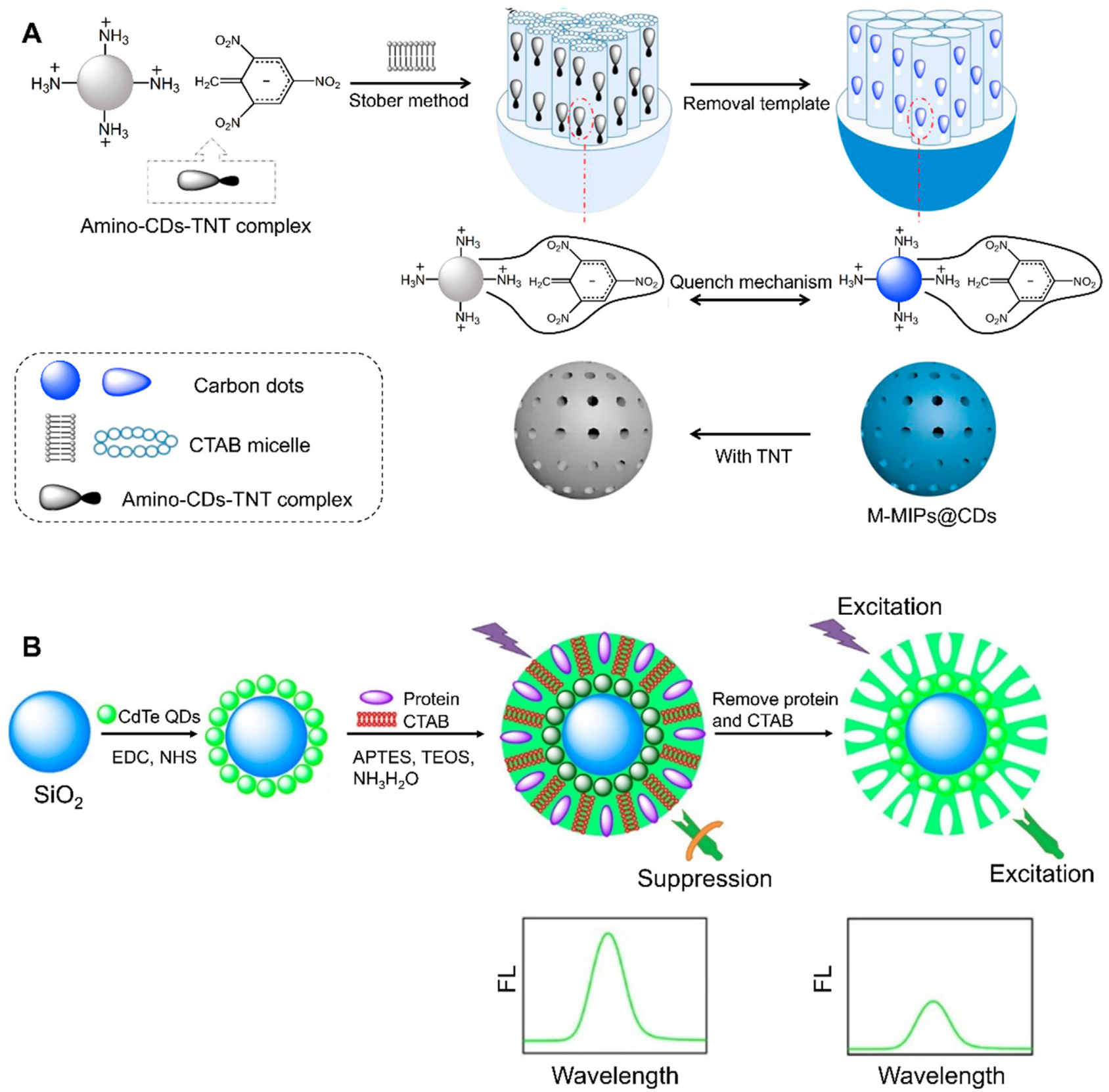

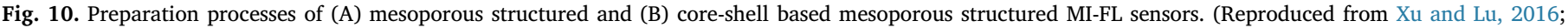
Zhang et al., 2015a, respectively).

was enhanced upon interaction with the template analyte and then lightened DBD-F through a FRET process; benefitting from the resolution of the FRET system, the plural fluorescent reporter had a binding constant of $2.72 \times 10^{-4} \mathrm{~mol} \mathrm{~L}^{-1}$, approximately twice the value of the single fluorescent reporter.

As mentioned above, PIMs of common MIPs have realized the fluorescence background lowering and in-cavity signal transduction. Furthermore, no interfering reagents (i.e., fluorescent materials) participating in the molecular imprinting procedure can also avoid the negative impact on the sensing properties.

\subsection{Ratiometric fluorescence detection}

Generally, fluorescence spectra scanning is compromised by a variety of analyte-independent factors, such as the local concentration of the fluorescent sensor, background interference, fluctuation of instrumental and operational parameters, and photobleaching, etc. Moreover, these single-emission sensors can only exhibit the variation of fluorescence brightness with analytes, which greatly limits their qualitative and quantitative capabilities. Hence, the ratiometric fluorescence technique has come into service for constructing MI-FL sensors, which usually contains two fluorescence substances of the target sensitive fluorescence dye and reference dye. Once excited by one single wavelength, the target sensitive fluorescence dye's fluorescence properties would change for analytes identification, while the reference dye is not affected by analytes, and then, based on the intensity ratio of two emission peaks ( $I_{\text {target sensitive dye }} / I_{\text {reference dye }}$ ), the ratiometric MI-FL sensors can realize the self-referencing effect to eliminate external interference and provide profuse color evolution for visual detection of target analytes.

Xu's group (Xu and Lu, 2015a, 2015b; Lu and Xu, 2017) has methodically presented the development of ratiometric MI-FL sensors. 


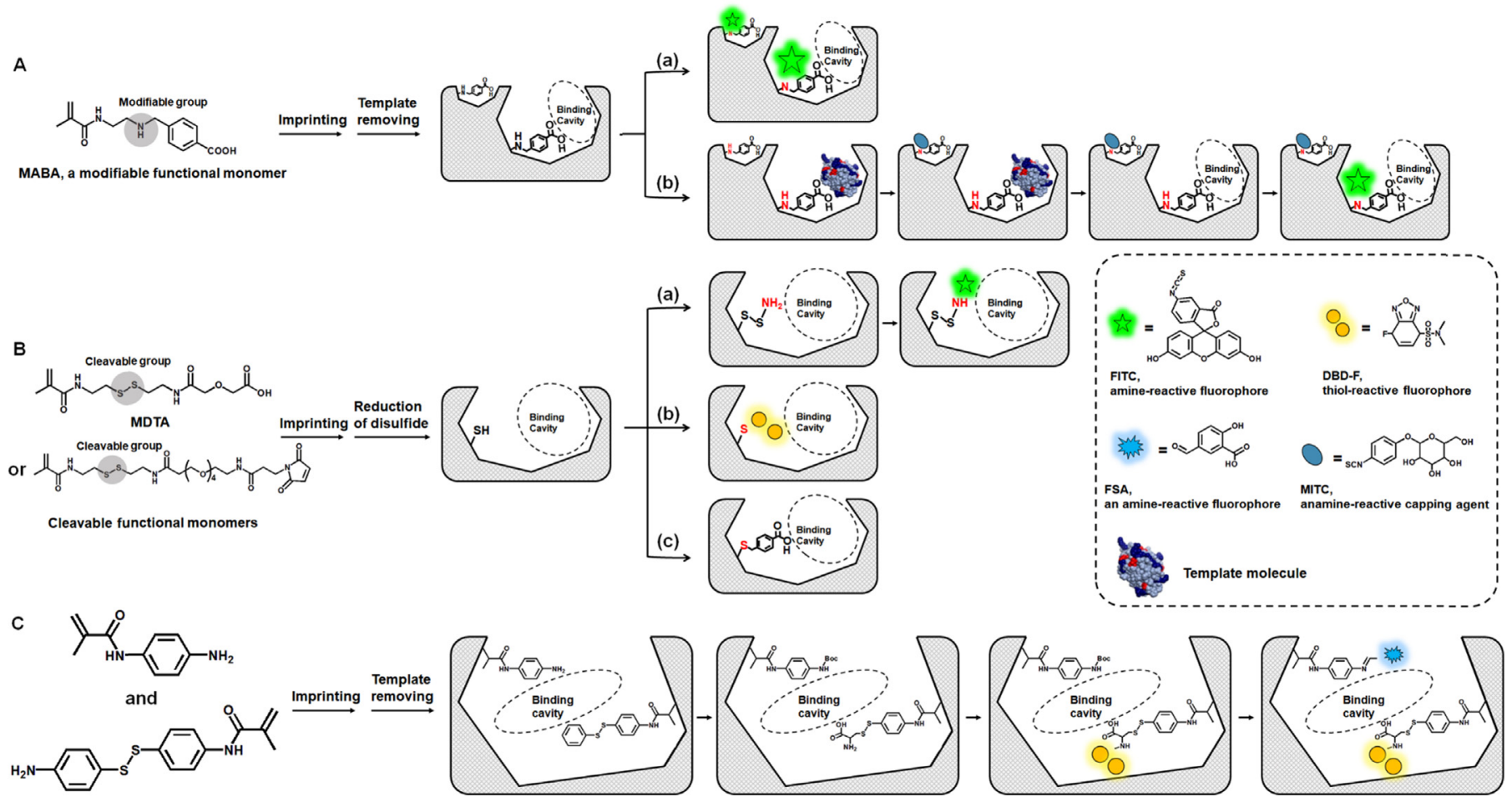

Fig. 11. PIMs on (A) directly modifiable functional monomers (Reproduced from Sunayama et al., 2010, 2014, respectively), (B) cleavable functional monomers (Reproduced from Sunayama and Takeuchi, 2014; Suga et al., 2013; Zhao et al., 2017, respectively) and (C) both modifiable and cleavable functional monomers (Adapted from Sunayama et al., 2016).

As illustrated in Fig. 12A, Xu and Lu (2015b) pioneered the ratiometric MI-FL sensor for TNT detection, with red CdTe QDs (reference dye) embedded Si-NPs as the core, TNP as a dummy template, CTAB as a template of mesoporous structure and green CdTe QD as a TNT sensitive fluorescence dye via a sol-gel procedure (namely, r-QD@SiO ${ }_{2} @ g$ QD@mMIP). Before rebinding TNP, yellow-green fluorescence was observed with dual emission peaks centered at $540 \mathrm{~nm}$ (green QDs) and $640 \mathrm{~nm}$ (red QDs); the rebound TNT quenched the green QDs in the imprinting shell via electron-transfer-induced resonance energy transfer, while the fluorescence intensity of red QDs kept constant with the protection of silica shell. Hence, fluorescence color changed from yellow-green to red-orange obviously, facilitating the visual detection of TNT from 50 to $600 \mathrm{nmol} \mathrm{L}^{-1}$ (LOD: $15 \mathrm{nmol} \mathrm{L}^{-1}$ ). Additionally, compared with dual-emission MIPs, the color change of single fluorescence quenching of the green QDs was hard to observe, and the linear range was narrow; compared with the non-mesoporous one, the sensitivity of the mesoporous one was largely improved with LOD decreasing from $\mu \mathrm{mol} \mathrm{L}{ }^{-1}$ to $\mathrm{nmol} \mathrm{L}^{-1}$. Inspired from the pioneering work, CdTe@ $\mathrm{SiO}_{2} @$ APTES-NBD@MIPs (Wang et al., 2016b), CdTe@SiO ${ }_{2} @ 2$-acrylamide-6-methoxybenzothiazole-mMIPs (Li et al., 2017b) and CQDs@ $\mathrm{SiO}_{2} @ \mathrm{CdTe} / \mathrm{CdS} @$ MIPs (Amjadi and Jalili, 2017) sequentially appeared, for 2,4-D, 2,4,6-trinitrophenol and diniconazole detection via electron and energy transfer, respectively. Obviously, it is preferred to employ QDs as a reference dye in a ratiometric MI-FL sensor, because QDs can be excited by the excited light of target sensitive fluorescence dyes owing to their broad excitation spectra. Beyond that, sharp emission profile of QDs is in favor of its separation from target sensitive dyes.

However, core-shell ratiometric MI-FL sensors are always prepared in at least two steps, which are time-consuming and hard to control: (i) embedding reference dyes into silica nanoparticle for blocking interaction with target analytes; (ii) imprinting on the surface of silica nanoparticles. Hence, one-pot method, i.e., incorporating two fluorescence dyes into the sensor by one synthesis procedure, is developed to simplify the synthesis process, shorten the experimental period, and meanwhile maintain the sensitivity of ratiometric fluorescence sensors. As shown in Fig. 12B, Xu and Lu (2015a) prepared a mesoporous MI-FL sensor to detect melamine by one-step polymerizing CdTe QDs (target sensitive dye, emission at $540 \mathrm{~nm}$ ) and hematoporphyrin (reference dye, emission at $620 \mathrm{~nm}$ ). The obtained ratiometric MI-FL sensor showed fluorescence quenching at $540 \mathrm{~nm}$ when it rebound melamine based on charge transfer mechanism, without fluorescence change of hematoporphyrin (Fig. 12B(b)). The ratiometric MI-FL sensor successfully realized visual signal output from yellow-green to red upon melamine changing from $100 \mathrm{nmol} \mathrm{L}^{-1}$ to $800 \mathrm{nmol} \mathrm{L}^{-1}$, with LOD down to $38 \mathrm{nmol} \mathrm{L}^{-1}$. A similar comparison of the single fluorescence sensor and non-mesoporous structured sensor was conducted, and the preponderance in the linear range, LOD and color change was conspicuous. Meanwhile, Li et al. (2016d) synthesized a similar ratiometric MI-FL sensor for paranitrophenol detection, using $\mathrm{Eu}^{+}$-activated yttrium orthovanadate $\left(\mathrm{YVO}_{4}: \mathrm{Eu}^{3+}\right)$ as reference dye and CQDs as target sensitive dye to detect 4-nitrophenol through two possible pathways of FRET and PET. Specifically, attention should be paid to the doping ratio of the two dyes: when the proportion of target sensitive dye was excessive, the fluorescence of reference dye would be masked and the selfcorrection effect weakened; conversely, when proportion of reference dye was immoderate, only a small relative change of target sensitive fluorescence dye upon target recognition would be observed to show narrow color changes. Wang et al. (2018b) proposed a novel method to avoid the time-consuming ratio optimization by separating reference dye from the ratiometric MIPs, i.e., preparing reference r-CdTe@SiO and target-sensitive Si-NP@g-QD@MIPs respectively, and further obtaining the desired color change with the targeted molecule added by simply mixing the two dyes at an appropriate ratio.

Although the one-pot method can effectively simplify the synthetic process, the condition optimization such as coupling or chemical modification is complicated in the construction process. $\mathrm{Lu}$ and $\mathrm{Xu}$ (2017) conducted further work for the above problems: designed the dual-emission nanoparticles (D-NPs) through seed growth co-microwave method with AuNPs as seeds and glucose as the precursor of 
A

A

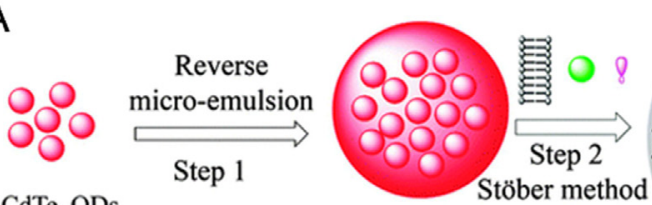
CdTe QDs

Step 1

QDs@SiO

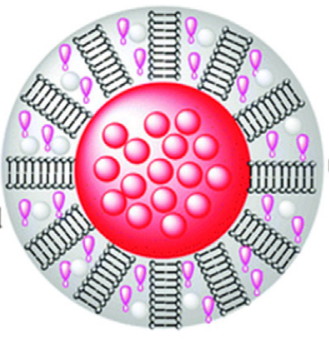

QDs@SiO $\mathrm{SiO}_{2} @ \mathrm{SiO}_{2}$
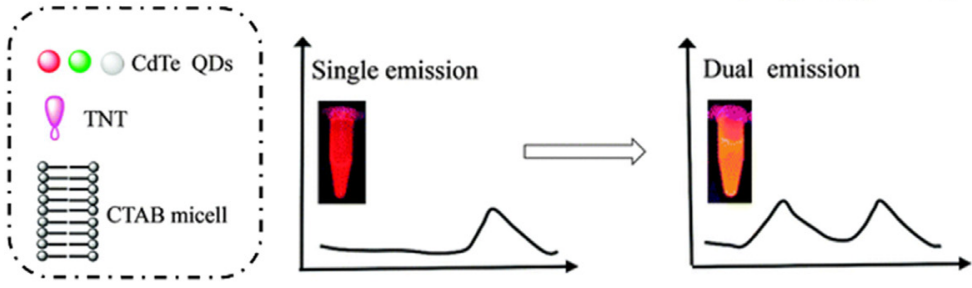

Removal

template

Step 3

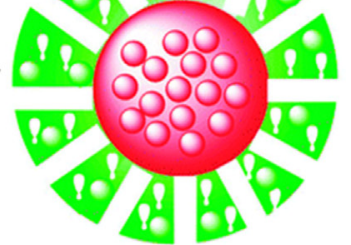

Imprinted QDs@SiO $\mathrm{Sim}_{2} \mathrm{SiO}_{2}$

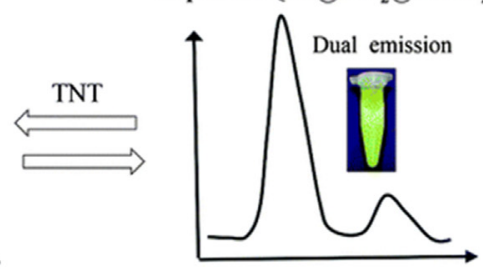

B

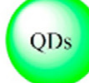

00

:ैâs Stöber method
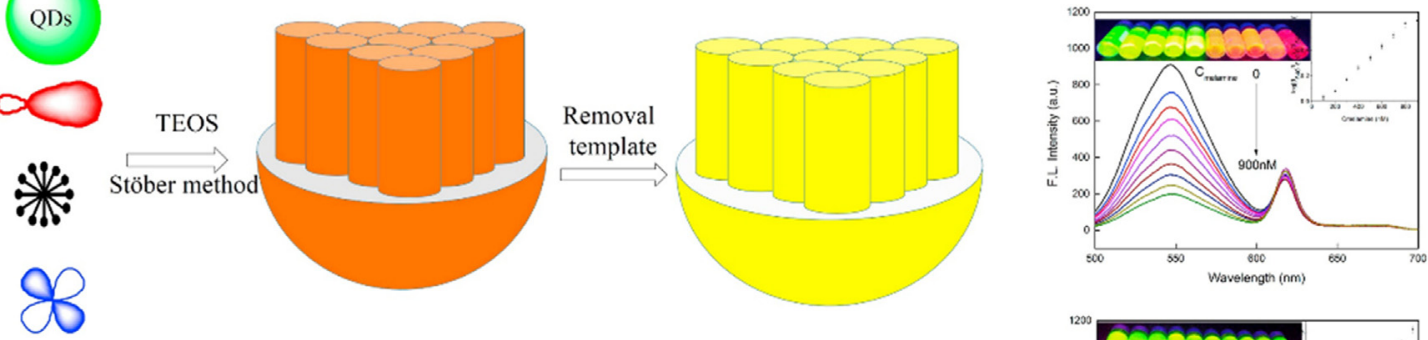

Template-functional monomer
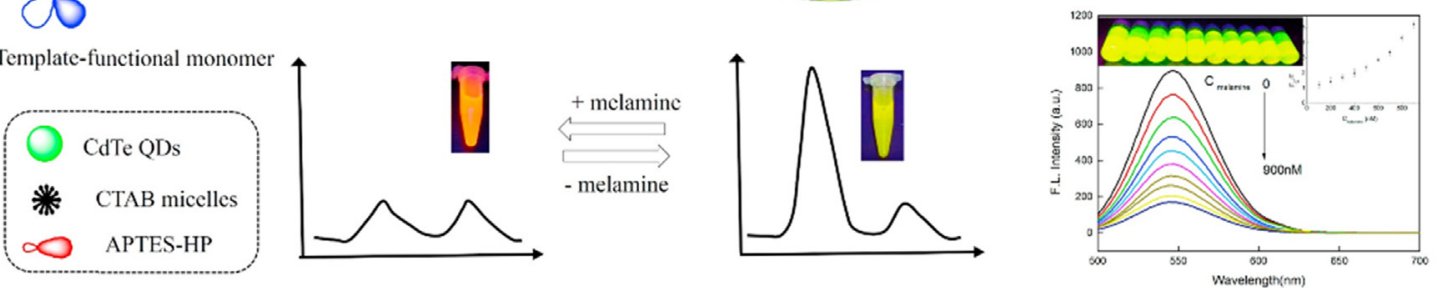

C
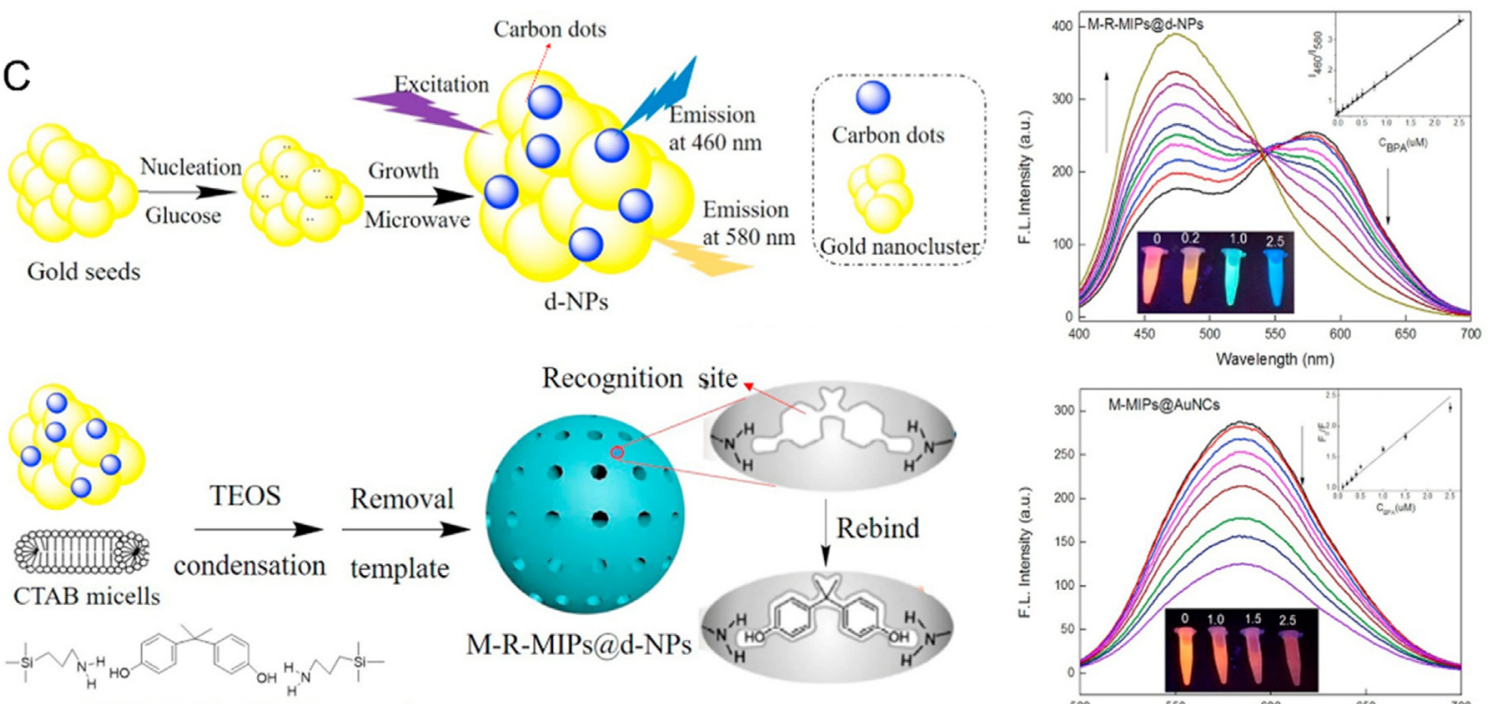

Recognition site,

APTES-BPA-APTES complex

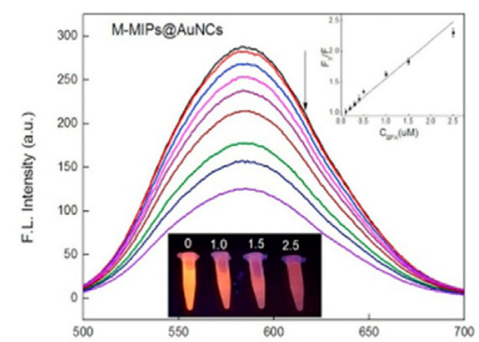

Wavelength (nm)

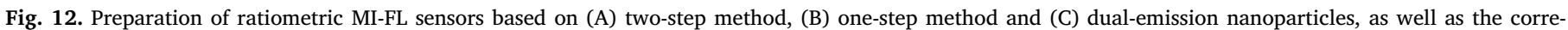
sponding fluorescence color change upon target analytes rebinding. (Reproduced from Xu and Lu, 2015b, 2015a; Lu and Xu, 2017, respectively). 
CQDs, and then synthesized a ratiometric MI-FL sensor using the D-NPs in the presence of BPA and CTAB, as described in Fig. 12C. Meisenheimer complex formed between the rebound BPA and amino groups of AuNCs, making fluorescence quenching of AuNCs at $580 \mathrm{~nm}$, and thereby, the fluorescence emission of CQDs at $460 \mathrm{~nm}$ increased because the FRET from CQDs to AuNCs was partly blocked. Based on the change in $I_{460} / I_{580}$, the fluorescence color changed from orange to green with BPA concentration ranging from $100 \mathrm{nmol} \mathrm{L}^{-1}$ to $2.5 \mu \mathrm{mol} \mathrm{L}$ ${ }^{1}$, easy to be observed by the naked eye. Compared with the single fluorescence sensor, the higher sensitivity and more obvious color evolution were clearly demonstrated.

Interestingly, different from the above to embed two external fluorescence signals into the MIPs, Wang et al. (2016b) developed a ratiometric MI-FL sensor by introducing only an external fluorescence dye of NBD, and the internal fluorescence source came from the target template of phycocyanin itself. FRET from APTES-NBD conjugates to the rebound phycocyanin resulted in fluorescence quenching of APTESNBD conjugates and fluorescence enhancing of phycocyanin. In addition, the exciting light of APTES-NBD conjugates could not make phycocyanin excited, avoiding the fluorescence generation of phycocyanin out of recognition sites. This work makes great sense for visual and sensitive detection of intrinsically fluorescence-active analytes, for instance, Rhodamine B (Li et al., 2017c) and doxorubicin (Xu et al., 2017).

To sum up, the evolution from the two-step method to the one-pot approach and the further D-NP-based process makes for simplifying the synthetic process. More efforts are deserved to be devoted to the reference built-in MI-FL sensors.

\section{MI-FL sensor based microdevices}

Developing MI-FL sensor composited microdevices is promising for realizing reliable point-of-care testing and in-field diagnosing within a few minutes, which is of great significance for rudimentary frontline screening. The three main MI-FL sensor based microdevices of micromotors, test strips and microfluidics are summarized.

\subsection{Micromotor}

Inspired from biological motors in eukaryotic cells that can convert chemical energy into mechanical work, some artificial motors at the nano- and microscale are designed to perform specific mechanical movements (e.g., rotation, shuttling, delivery and collective behavior) in response to specific energy input. According to the type of energy input, the nano- and micromotors are principally characterized as catalytic, photodriven, magnetically, electrically, and ultrasonically driven nano- and micromotors. Once incorporated with molecular imprinting and fluorescence technology (Orozco et al., 2013; Zhang et al., 2015b), these nano- and micromotors can accordingly provide autonomous and selective predator-prey or enrichment behavior during the tailor-made self-propelled movements even under extreme conditions, accompanying specific optical phenomenon. For instance, Orozco et al. (2013) designed a FITC-labeled avidin (Av-FITC) imprinted micromotor as illustrated in Fig. 13: (a, b) Av-FITC template was first adsorbed in the inner walls of the micropores of a polycarbonate membrane; (c) poly (3,4-ethylenedioxythiophene), Pt, Ni, and Pt metallic layers were sequentially electrodeposited on the inner walls; and (e) the polycarbonate membrane was dissolved and Av-FITC was removed to finally obtain (d) the Av-FITC imprinted tubular micromotor. In test solution, the inner Pt layer catalytically oxidized hydrogen peroxide and generated oxygen bubbles, propelling the micromotor in a straight line (Fig. 13f). At the same time, the micromotor offered specific Av-FITC recognition, capture and transport, permitting the real-time optical visualization based on fluorescence intensity change. Inspired from the above work, our group (Zhang et al., 2015b) developed a phycocyaninimprinted tubular micromotor which had an autonomously straight or spiral motion trajectory, guided by an additional magnetic field.

Apart from hydrogen peroxide, actually, water (Gao et al., 2012; Wu et al., 2015c), acids/bases (Dey et al., 2013; Li et al., 2017d) and dual-/ multi-fuel (Gao et al., 2013) can also provide energy for the catalytic micromotors. MIPs- and fluorescence-based micromotors can make the best use of these different fuels, or even other physical resources. Additionally, reducing the scale of micromotors to nanoscale may be the tendency for sensitivity improvement.

Evidently, ordinary MI-FL sensors and micromotor-based devices are mostly applied in the aqueous phase. Realizing the liquid phase being transferred to the solid support can improve the portability and operability.

\subsection{Test strip}

One form of solid-phase MI-FL microdevices is a test strip, which can be simply proposed by grafting MI-FL sensors on a proper substrate to fully integrate their respective advantages but is rarely reported (Chen, 2017; Zhang et al., 2016). Taking a polyimide-based test strip as an example, Chen (2017) invented a test strip for tetracycline detection by bonding MIP-QDs composites to methacrylated polyimide (PI) substrates, as illustrated in Fig. 14. MIPs were first coupled onto QD surface to form MIPs-QD composites with unreacted methacrylate residues (Fig. 14A); methacrylate groups were driven onto the pretreated (aminolysis or alkaline hydrolysis) PI by three different methacrylate reagents (Fig. 14B); and MIPs-QD composites were bound to the methacrylated PI on a hot plate at $65^{\circ} \mathrm{C}$ through a radical-initiated polymerization, with the subsequent tetracycline removal (Fig. 14C). Through dropping tetracycline on the test strip and measuring the quenching intensity, the concentration of tetracycline down to

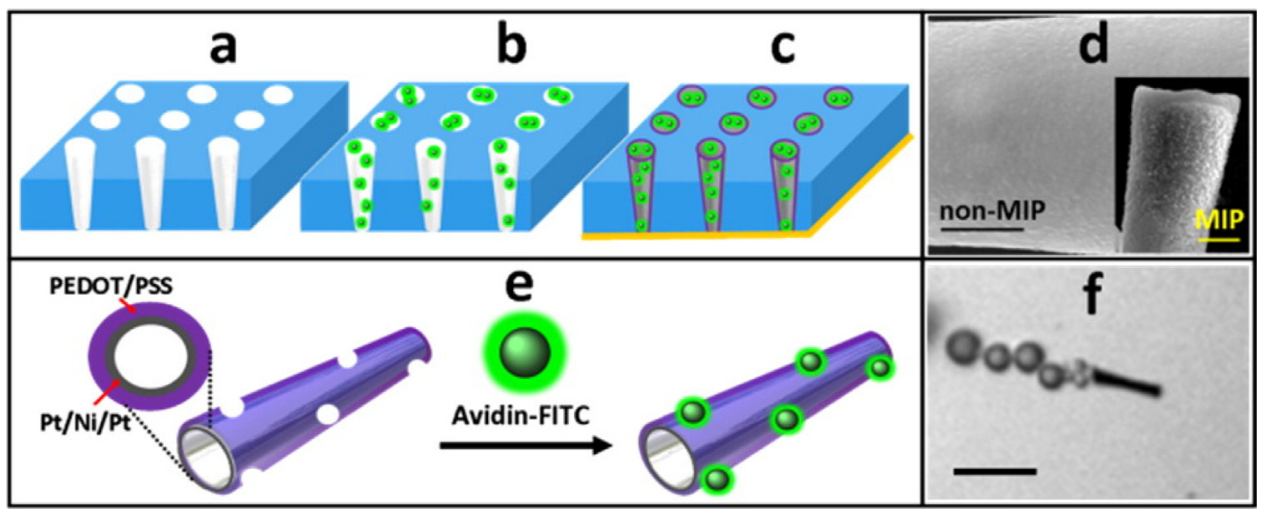

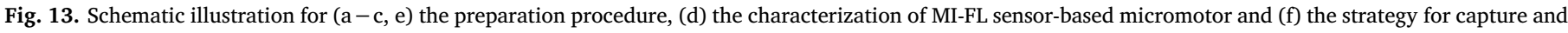
transport of the target protein (Adapted from Orozco et al., 2013). 

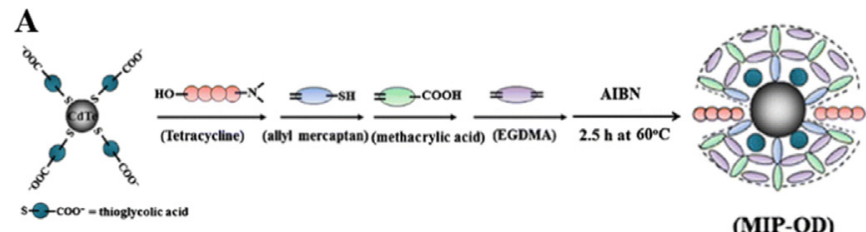

$\mathbf{B}$
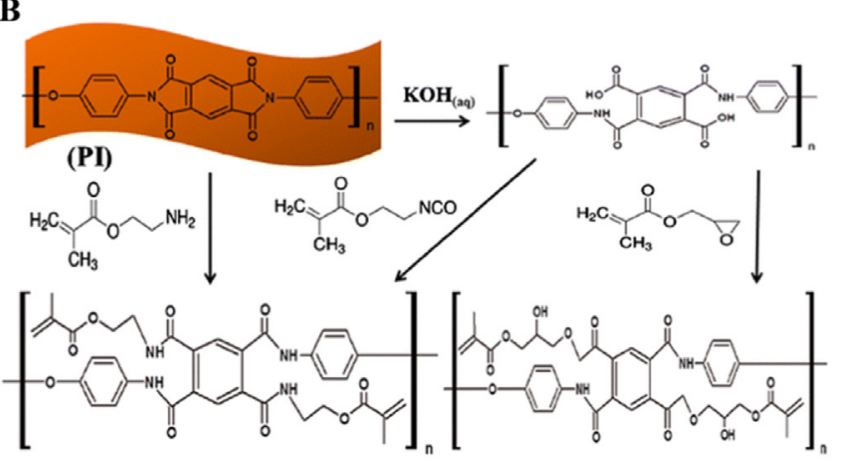

(Am-PI)

(Iso-PI)

(Gly-PI)

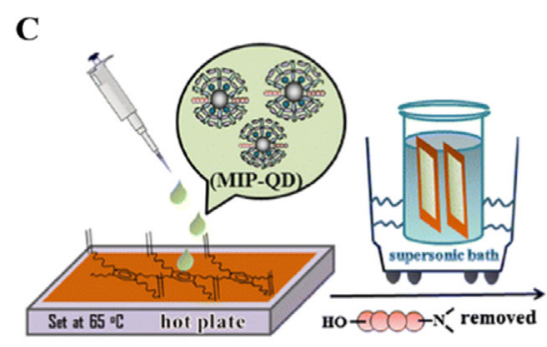

(Am-PI; Iso-PI; Gly-PI)

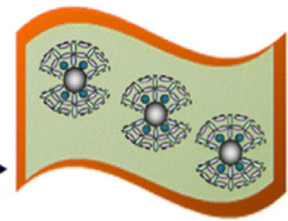

(Am-MIP; Iso-MIP; Gly-MIP)

Fig. 14. Preparation procedures of (a) MIP-QD composites, (b) methacrylated polyimide substrates (PI), and (c) MIP-QDs on PI with stripping of the tetracycline template. (Reproduced from Chen 2017).

$8.8 \mu \mathrm{mol} \mathrm{L}{ }^{-1}$ could be detected.

\subsection{Microfluidic}

Microfluidics can provide the other solid matrix for MI-FL materials. Microfluidics typically comprise microfabricated structures for precise manipulation of liquids, with femtoliter- to nanoliter-scale volume capacity for their cross section in the $1-500 \mu \mathrm{m}$ range. Small distances of

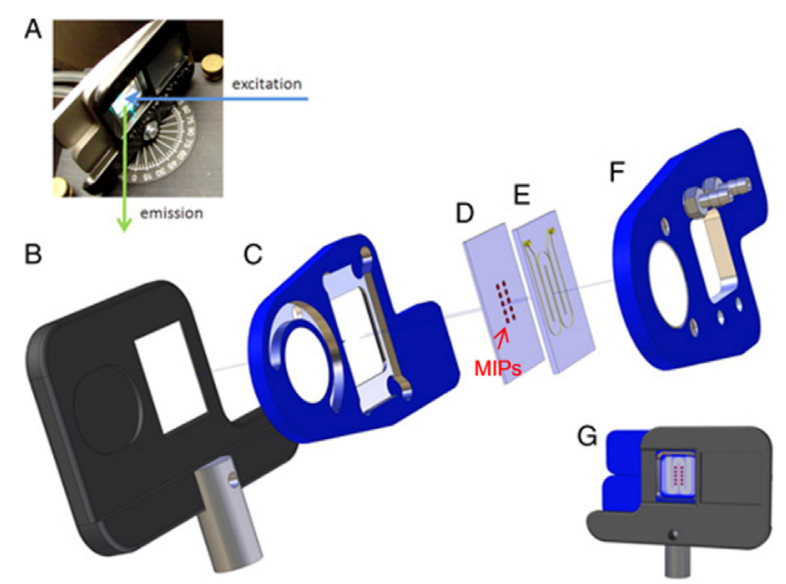

Fig. 15. Molecular imprinting based microfluidic chip holder with chip cuvette and holder for fluorescence measurements in a spectrofluorometer. (Reproduced from Harz et al., 2011). microfluidics lead to fast interaction; small channel dimensions bring about small sample volumes; and reduced dimensions create portable devices for on-site monitoring (Nge et al., 2013). Materials for microfluidics preparation can be simply organized into three main categories: inorganic (Iliescu et al., 2012), polymeric (Effenhauser et al., 1997; Gao et al., 2014) and paper materials.

Taking quartz-based material as an example, Harz et al. (2011) designed a MIPs-immobilized microfluidic measurement setup with fluorescence detection for dansyl-L-phenylalanine measurement down to $0.5 \mathrm{mmol} \mathrm{L}^{-1}$. As illustrated in Fig. 15, the prepared MIPs particles were first immobilized on the surface of quartz substance via a molecular glue (Fig. 15D), followed with being bounded to a microstructured chip, where two holes acted as inlet and outlet, as well as the meander guided the flow over the imprinting surface (Fig. 15E). Then, a chip holder (Fig. 15C and F) with $\mathrm{O}$ rings was used to connect the microfluidic chip with the tube for fluid transport, and the holder had an optical window for fluorescence measurement and two magnetically coupled parts to easily change the chip. The entire assembled setup is shown in Fig. 15G. For dansyl-L-phenylalanine measurement, the sample was transported into the chip by syringe pumps, and then, the fluorescence intensity was measured using a spectrofluorometer, where the excitation angle was empirically designed to be $23^{\circ}$ to obtain the highest signal-to-noise ratio with the lowest background (Fig. 15A).

Moreover, paper-based microfluidic analytical devices, pioneered by Whitesides' group (Martinez et al., 2007), are also promising for being introduced into MI-FL sensors. Recently, our group (Li et al., 2017e) has fabricated a three-dimensional origami paper-based microfluidic device based on the paper@QDs@PC-MIPs (namely, PQPMIPs) preparation. Briefly, the hydroxyl-active filter paper was consecutively grafted with amino groups, CdTe QDs, and phycocyaninimprinting layer to prepare PQP-MIPs. Meanwhile, a three-dimensional origami paper was patterned by wax to define hydrophobic areas (green, yellow and blue part) that can provide spatial control for fluids. Then, the obtained PQP-MIPs component was attached to the pink part of the bottom layer, followed by assembling with clamps (left part of Fig. 16). For phycocyanin measurement, the test solution was added to the sample reservoir and then was wicked into the detection area under a hydrophobic barrier and capillary force. After filtered from the top layer into the bottom layer, phycocyanin can be rebound by the imprinted cavities and form Meisenheimer complex with CdTe QD to cause fluorescence quenching (right part of Fig. 16). Furthermore, based on ion imprinting technology (Fu et al., 2015), our group (Qi et al., 2017) has proposed another paper-based microfluidic chip device with "Y"-type channel for multiplexed fluorescence detection of $\mathrm{Cu}^{2+}$ and $\mathrm{Hg}^{2+}$ ions. Moreover, both paper-based microfluidics could realize the simple filter function for dirty aqueous samples.

Overall, these microdevices could detect target analytes specifically and conveniently, particularly the solid-phase test strips and microfluidics. What is more, these sensing procedures are simplified to allow untrained people to readily perform analysis but without learning about the sophisticated sensing principle involved. However, most of these microdevices are limited for fluorescent or fluorescence-labeled analytes without prominent sensitivity. If more new construction methods toward nonfluorescent targets are integrated, these microdevices will have tremendous global markets. Additionally, the demand for common fluorescence spectrometers to distinguish minimal spectral changes and interpret molecular interactions hinders the portability (Harz et al., 2011). Hence, apart from developing the miniaturized optical detectors with high optical resolution, the alternative is to design sensing devices that can output profuse color upon analyte rebinding for visual observation, for example, introducing the ratiometric fluorescence technology.

\section{Conclusions and perspectives}

In this review, state-of-arts of MI-FL sensors are summarized, 

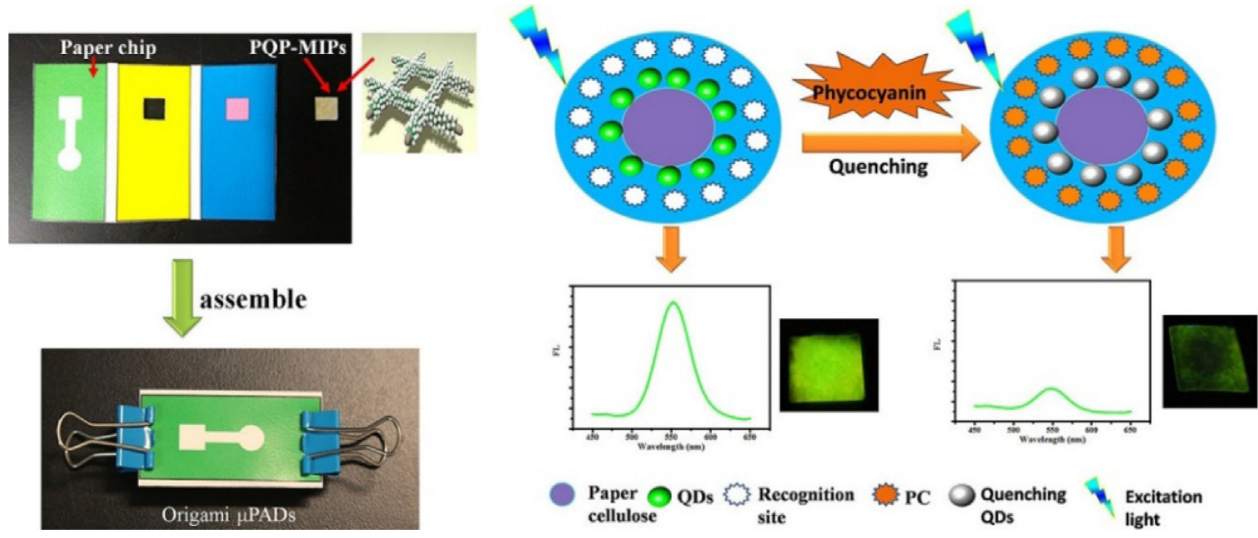

Fig. 16. Preparation procedure and sensing principle of the MI-FL sensor-based three-dimensional origami paper microfluidic device. (Adapted from Li et al., 2017e).

placing particular emphasis on the general and novel construction strategies and the signal transduction in sensing procedure, as well as the portable MI-FL sensor based microdevices. For their unique advances in sensitivity and selectivity, there is no doubt that MI-FL sensors will grow at an even faster pace. The challenges and perspectives may focus on the following areas: (i) development of MI-FL sensors for viruses and bacteria on the basic of micro- and macromolecule imprinting technique; (ii) development of novel fluorescent materials taking account of photo performance, economic and environmental friendliness; (iii) further overcoming various defects associated with MIPs (e.g., mass transfer resistance and inaccessible recognition sites) and fluorescence (e.g., high background and analyte-independent disturbance in fluorescence measurement); (iv) exploring the signal transduction mechanisms in MI-FL sensor and devoting more mechanisms into sensor design besides PET and FRET; (v) deeper research in MI-FL sensor based portable devices and developing them into commercialization rather than a proof-of-concept demonstration; (vi) rational design of MI-FL sensors with high-throughput assays to meet the increasing test requirement.

\section{Acknowledgments}

This work was financially supported by the National Natural Science Foundation of China (21667018, 21477160, 31660482, 41776110, 21575159), and the Natural Science Foundation of Shandong Province of China (ZR2016BL25).

\section{Conflict of interest}

The authors declare no competing financial interest.

\section{References}

Amjadi, M., Jalili, R., 2017. Molecularly imprinted mesoporous silica embedded with carbon dots and semiconductor quantum dots as a ratiometric fluorescent sensor for diniconazole. Biosens. Bioelectron. 96 (2017), 121-126.

Andersson, L.I., Mandenius, C.F., Mosbach, K., 1988. Studies on guest selective molecular recognition on an octadecyl silylated silicon surface using ellipsometry. Tetrahedron Lett. 29, 5437-5440.

Auzel, F., 2004. Upconversion and anti-stokes processes with $\mathrm{f}$ and $\mathrm{d}$ ions in solids. Chem. Rev. 104, 139-174.

Awino, J.K., Zhao, Y., 2014. Molecularly imprinted nanoparticles as tailor-made sensors for small fluorescent molecules. Chem. Commun. 50, 5752-5755.

Boyer, J.C., Manseau, M.P., Murray, J.I., Veggel, F.C.J.M., 2010. Surface modification of upconverting $\mathrm{NaYF}_{4}$ nanoparticles with PEG - phosphate ligands for NIR (800 nm) biolabeling within the biological window. Langmuir 26, 1157-1164.

Brus, L.E., 1983. A simple model for the ionization potential, electron affinity, and aqueous redox potentials of small semiconductor crystallites. J. Chem. Phys. 79, 5566-5571.

Carrasco, S., Canalejas-Tejero, V., Navarro-Villoslada, F., Barrios, C.A., Moreno-Bondi, M.C., 2014. Cross-linkable linear copolymer with double functionality: resist for electron beam nanolithography and molecular imprinting. J. Mater. Chem. C 2, 1400-1403.
Chantada-Vázquez, M.P., Sánchez-González, J., Peña-Vázquez, E., Tabernero, M.J., Bermejo, A.M., Bermejo-Barrera, P., Moreda-Piñeiro, A., 2016. Synthesis and characterization of novel molecularly imprinted polymer-coated Mn-doped ZnS quantum dots for specific fluorescent recognition of cocaine. Biosens. Bioelectron. 75, 213-221.

Chao, M.R., Hu, C.W., Chen, J.L., 2014. Comparative syntheses of tetracycline-imprinted polymeric silicate and acrylate on CdTe quantum dots as fluorescent sensors. Biosens. Bioelectron. 61, 471-477.

Chen, K., He, R., Luo, X., Qin, P., Tan, L., Tang, Y., Yang, Z., 2017a. A fluorescent glycosyl-imprinted polymer for $\mathrm{pH}$ and temperature regulated sensing of target glycopeptide antibiotic. Biosens. Bioelectron. 94, 609-615.

Chen, D., Gao, H., Chen, X., Fang, G., Yuan, S., Yuan, Y., 2017b. Excitation-independent dual-color carbon dots: surface-state controlling and solid-state lighting. ACS Photonics 4, 2352-2358.

Chen, J.L., 2017. Determination of tetracycline using imprinted polymethacrylates along with fluorescent CdTe quantum dots on plastic substrates. Microchim. Acta 5 , 1335-1343.

Chen, L., Xu, S., Li, J., 2011. Recent advances in molecular imprinting technology: current status, challenges and highlighted applications. Chem. Soc. Rev. 40, 2922-2942.

Chen, L., Wang, X., Lu, W., Wu, X., Li, J., 2016. Molecular imprinting: perspectives and applications. Chem. Soc. Rev. 45, 2137-2211.

Chen, Z., Álvarez-Pérez, M., Navarro-Villoslada, F., Moreno-Bondi, M.C., Orellana, G., 2014. Fluorescent sensing of "quat" herbicides with a multifunctional pyrene-labeled monomer and molecular imprinting. Sens. Actuators B: Chem. 191, 137-142.

Deng, C., Zhong, Y., He, Y., Ge, Y., Song, G., 2016. Selective determination of trace bisphenol a using molecularly imprinted silica nanoparticles containing quenchable fluorescent silver nanoclusters. Microchim. Acta 183, 431-439.

Dey, K.K., Bhandari, S., Bandyopadhyay, D., Basu, S., Chattopadhyay, A., 2013. The pH taxis of an intelligent catalytic microbot. Small 9, 1916-1920.

Effenhauser, C.S., Bruin, G.J.M., Paulus, A., Ehrat, M., 1997. Integrated capillary electrophoresis on flexible silicone microdevices: analysis of DNA restriction fragments and detection of single DNA molecules on microchips. Anal. Chem. 69, 3451-3457.

Efros, A.L., Efros, A.L., 1982. Interband light absorption in semiconductor spheres. Sov. Phys. Semicond. 16, 772-775.

El Gohary, N.A., Madbouly, A., El Nashara, R.M., Mizaikoff, B., 2015. Synthesis and application of a molecularly imprinted polymer for the voltammetric determination of famciclovir. Biosens. Bioelectron. 65, 108-114.

EL-Sharif, H.F., Aizawa, H., Reddy, S.M., 2015. Spectroscopic and quartz crystal microbalance (QCM) characterisation of protein-based MIPs. Sens. Actuators B: Chem. 206, $239-245$.

Feng, L., Tan, L., Li, H., Xu, Z., Shen, G., Tang, Y., 2015. Selective fluorescent sensing of $\alpha$ amanitin in serum using carbon quantum dots-embedded specificity determinant imprinted polymers. Biosens. Bioelectron. 69, 265-271.

Förster, T., 1948. Zwischenmolekulare Energiewanderung und Fluoreszenz. Ann. Phys. 437, 55-75.

Fu, J., Chen, L., Li, J., Zhang, Z., 2015. Current status and challenges of ion imprinting. J. Mater. Chem. A 3, 13598-13627.

Gao, K., Li, L., He, L., Hinkle, K., Wu, Y., Ma, J., Chang, L., Zhao, X., Perez, D.G., Eckardt, S., Mclaughlin, J., Liu, B., Farson, D.F., Lee, L.J., 2014. Nanochannel-microchannel array based nanoelectroporation system for precise gene transfection. Small 10, 1015-1023.

Gao, W., Pei, A., Wang, J., 2012. Water-driven micromotors. ACS Nano 6, 8432-8438.

Gao, W., D'Agostino, M., Garcia-Gradilla, V., Orozco, J., Wang, J., 2013. Multi-fuel driven Janus micromotors. Small 9, 467-471.

Guo, T., Deng, Q., Fang, G., Liu, C., Huang, X., Wang, S., 2015. Molecularly imprinted upconversion nanoparticles for highly selective and sensitive sensing of Cytochrome c. Biosens. Bioelectron. 74, 498-503.

Guo, T., Deng, Q., Fang, G., Gu, D., Yang, Y., Wang, S., 2016a. Upconversion fluorescence metal-organic frameworks thermo-sensitive imprinted polymer for enrichment and sensing protein. Biosens. Bioelectron. 79, 341-346.

Guo, T., Deng, Q., Fang, G., Yun, Y., Hu, Y., Wang, S., 2016b. A double responsive smart upconversion fluorescence sensing material for glycoprotein. Biosens. Bioelectron. 85, 596-602. 
Harz, S., Schimmelpfennig, M., Bui, B.T.S., Marchyk, N., Haupt, K., Feller, K.H., 2011. Fluorescence optical spectrally resolved sensor based on molecularly imprinted polymers and microfluidics. Eng. Life Sci. 11, 559-565.

Huy, B.T., Seo, M.H., Zhang, X., Lee, Y.I., 2014. Selective optosensing of clenbuterol and melamine using molecularly imprinted polymer-capped CdTe quantum dots. Biosens. Bioelectron. 57, 310-316.

Iliescu, C., Taylor, H., Avram, M., Miao, J., Franssila, S., 2012. A practical guide for the fabrication of microfluidic devices using glass and silicon. Biomicrofluidics 6, 16505.

Ivanova-Mitseva, P.K., Guerreiro, A., Piletska, E.V., Whitcombe, M.J., Zhou, Z., Mitsev, P.A., Davis, F., Piletsky, S.A., 2012. Cubic molecularly imprinted polymer nanoparticles with a fluorescent core. Angew. Chem. Int. Ed. 51, 5196-5199.

Jalili, R., Amjadi, M., 2018. Bio-inspired molecularly imprinted polymer-green emitting carbon dot composite for selective and sensitive detection of 3-nitrotyrosine as a biomarker. Sens. Actuators B: Chem. 255, 1072-1078.

Jia, M., Zhang, Z., Li, J., Shao, H., Chen, L., Yang, X., 2017. A molecular imprinting fluorescence sensor based on quantum dots and a mesoporous structure for selective and sensitive detection of 2,4-dichlorophenoxyacetic acid. Sens. Actuators B: Chem. 252, 934-943.

Johnson-White, B., Zeinali, M., Shaffer, K.M., Patterson Jr., C.H., Charles, P.T., Markowitz, M.A., 2007. Detection of organics using porphyrin embedded nanoporous organosilicas. Biosens. Bioelectron. 22, 1154-1162.

Kim, J.S., Quang, D.T., 2007. Calixarene-derived fluorescent probes. Chem. Rev. 107, 3780-3799.

Lakowicz, J.R., 2006. Principles of Fluorescence Spectroscopy, third ed. Springer, Boston, MA.

Lee, M.H., Thomas, J.L., Chen, Y.L., Lin, C.F., Tsai, H.H., Juang, Y.Z., Liu, B.D., Lin, H.Y., 2013. Optical sensing of urinary melatonin with molecularly imprinted poly(ethylene-co-vinyl alcohol) coated zinc oxide nanorod arrays. Biosens. Bioelectron. 47, 56-61.

Li, J., Zhang, Z., Xu, S., Chen, L., Zhou, N., Xiong, H., Peng, H., 2011a. Label-free colorimetric detection of trace cholesterol based on molecularly imprinted photonic hydrogels. J. Mater. Chem. 21, 19267-19274.

Li, Y., Dong, C., Chu, J., Qi, J., Li, X., 2011b. Surface molecular imprinting onto fluorescein-coated magnetic nanoparticles via reversible addition fragmentation chain transfer polymerization: a facile three-in-one system for recognition and separation of endocrine disrupting chemicals. Nanoscale 3, 280-287.

Li, D.Y., He, X.W., Chen, Y., Li, W.Y., Zhang, Y.K., 2013. Novel hybrid structure silica/ $\mathrm{CdTe} /$ molecularly imprinted polymer: synthesis, specific recognition, and quantitative fluorescence detection of bovine hemoglobin. ACS Appl. Mater. Interfaces 5, 12609-12616.

Li, D.Y., Qin, Y.P., Li, H.Y., He, X.W., Li, W.Y., Zhang, Y.K., 2015a. A “turn-on" fluorescent receptor for detecting tyrosine phosphopeptide using the surface imprinting procedure and the epitope approach. Biosens. Bioelectron. 66, 224-230.

Li, S., Yin, G., Zhang, Q., Li, C., Luo, J., Xu, Z., Qin, A., 2015b. Selective detection of fenaminosulf via a molecularly imprinted fluorescence switch and silver nano-film amplification. Biosens. Bioelectron. 71, 342-347.

Li, Q., Kamra, T., Ye, L., 2016a. A modular approach for assembling turn-on fluorescence sensors using molecularly imprinted nanoparticles. Chem. Commun. 52 12237-12240.

Li, D.Y., Zhang, X.M., Yan, Y.J., He, X.W., Li, W.Y., Zhang, Y.K., 2016b. Thermo-sensitive imprinted polymer embedded carbon dots using epitope approach. Biosens. Bioelectron. 79, 187-192.

Li, X.G., Zhang, F., Gao, Y., Zhou, Q.M., Zhao, Y., Li, Y., Huo, J.Z., Zhao, X.J., 2016c. Facile synthesis of red emitting 3-aminophenylboronic acid functionalized copper nanoclusters for rapid, selective and highly sensitive detection of glycoproteins. Biosens. Bioelectron. 86, 270-276.

Li, W., Zhang, H., Chen, S., Liu, Y., Zhuang, J., Lei, B., 2016d. Synthesis of molecularly imprinted carbon dot grafted $\mathrm{YVO}_{4}: \mathrm{Eu}^{3+}$ for the ratiometric fluorescent determination of paranitrophenol. Biosens. Bioelectron. 86, 706-713.

Li, H., Wei, X., Xu, Y., Lu, K., Zhang, Y., Yan, Y., Li, C., 2017b. A thin shell and "sunny shape" molecular imprinted fluorescence sensor in selective detection of trace level pesticides in river. J. Alloy. Compd. 705, 524-532.

Li, M., Liu, H., Ren, X., 2017b. Ratiometric fluorescence and mesoporous structured imprinting nanoparticles for rapid and sensitive detection 2,4,6-trinitrophenol. Biosens. Bioelectron. 89, 899-905.

Li, H., Li, N., Jiang, J., Chen, D., Xu, Q., Li, H., He, J., Lu, J., 2017a. Molecularly imprinted magnetic microparticles for the simultaneous detection and extraction of Rhodamine B. Sens. Actuators B: Chem. 246, 286-292.

Li, J., Angsantikul, P., Liu, W., Ávila, B.E.F., Thamphiwatana, S., Xu, M., Sandraz, E., Wang, X., Delezuk, J., Gao, W., Zhang, L., Wang, J., 2017d. Micromotors spontaneously neutralize gastric acid for pH-responsive payload release. Angew. Chem. Int. Ed. 56, 2156-2161.

Li, B., Zhang, Z., Qi, J., Zhou, N., Qin, S., Choo, J., Chen, L., 2017e. Quantum dot-based molecularly imprinted polymers on three-dimensional origami paper microfluidic chip for fluorescence detection of phycocyanin. ACS Sens. 2, 243-250.

Li, Z., Barnes, J.C., Bosoy, A., Stoddart, J.F., Zink, J.I., 2012. Mesoporous silica nanoparticles in biomedical applications. Chem. Soc. Rev. 41, 2590-2605.

Lim, S.Y., Shen, W., Gao, Z., 2015. Carbon quantum dots and their applications. Chem. Soc. Rev. 44, 362-381.

Lin, L., Rong, M., Luo, F., Chen, D., Wang, Y., Chen, X., 2014. Luminescent graphene quantum dots as new fluorescent materials for environmental and biological applications. Trac-Trend Anal. Chem. 54, 83-102.

Liu, C., Song, Z., Pan, J., Wei, X., Gao, L., Yan, Y., Li, L., Wang, J., Chen, R., Dai, J., Yu, P., 2013. Molecular imprinting in fluorescent particle stabilized pickering emulsion for selective and sensitive optosensing of $\lambda$-cyhalothrin. J. Phys. Chem. C 117, 10445-10453.
Liu, C., Song, Z., Pan, J., Yan, Y., Cao, Z., Wei, X., Gao, L., Wang, J., Dai, J., Meng, M., Yu, P., 2014a. A simple and sensitive surface molecularly imprinted polymers based fluorescence sensor for detection of $\lambda$-Cyhalothrin. Talanta 125, 14-23.

Liu, H., Fang, G., Wang, S., 2014b. Molecularly imprinted optosensing material based on hydrophobic CdSe quantum dots via a reverse microemulsion for specific recognition of ractopamine. Biosens. Bioelectron. 55, 127-132.

Liu, H., Chen, X., Mu, L., Wang, J., Sun, B., 2016. Application of quantum dot-molecularly imprinted polymer core-shell particles sensitized with graphene for optosensing of $N^{\varepsilon}$-carboxymethyllysine in dairy products. J. Agric. Food Chem. 64, 4801-4806.

Liu, H., Ni, T., Mu, L., Zhang, D., Wang, J., Wang, S., Sun, B., 2018. Sensitive detection of pyrraline with a molecularly imprinted sensor based on metal-organic frameworks and quantum dots. Sens. Actuators B: Chem. 256, 1038-1044.

Liu, X., Ren, J., Su, L., Gao, X., Tang, Y., Ma, T., Zhu, L., Li, J., 2017. Novel hybrid probe based on double recognition of aptamer-molecularly imprinted polymer grafted on upconversion nanoparticles for enrofloxacin sensing. Biosens. Bioelectron. 87, 203-208.

Liu, Z.C., Qi, J.W., Hu, C., Zhang, L., Song, W., Liang, R.P., Qiu, J.D., 2015. Cu nanoclusters-based ratiometric fluorescence probe for ratiometric and visualization detection of copper ions. Anal. Chim. Acta 895, 95-103.

Lu, H., Xu, S., 2017. Visualizing BPA by molecularly imprinted ratiometric fluorescence sensor based on dual emission nanoparticles. Biosens. Bioelectron. 92, 147-153.

Lu, X., Yang, Y., Zeng, Y., Li, L., Wu, X., 2018. Rapid and reliable determination of $p$ nitroaniline in wastewater by molecularly imprinted fluorescent polymeric ionic liquid microspheres. Biosens. Bioelectron. 99, 47-55.

Manju, S., Hari, P.R., Sreenivasan, K., 2010. Fluorescent molecularly imprinted polymer film binds glucose with a concomitant changes in fluorescence. Biosens. Bioelectron. 26, 894-897.

Martinez, A.W., Phillips, S.T., Butte, M.J., Whitesides, G.M., 2007. Patterned paper as a platform for inexpensive, low-volume, portable bioassays. Angew. Chem. Int. Ed. 46, 1318-1320.

Martinez-Manez, R., Sancenón, F., 2003. Fluorogenic and chromogenic chemosensors and reagents for anions. Chem. Rev. 103, 4419-4476.

Mehrzad-Samarin, M., Faridbod, F., Dezfuli, A.S., Ganjali, M.R., 2017. A novel metronidazole fluorescent nanosensor based on graphene quantum dots embedded silica molecularly imprinted polymer. Biosens. Bioelectron. 92, 618-623.

Miller, F.P., Vandome, A.F., Mcbrewster, J., 2010. Fluorophore. Alphascript Publishing.

Ming, W., Wang, X., Lu, W., Zhang, Z., Song, X., Li, J., Chen, L., 2017. Magnetic molecularly imprinted polymers for the fluorescent detection of trace 17ß-estradiol in environmental water. Sens. Actuators B: Chem. 238, 1309-1315.

Murase, N., Taniguchi, S., Takano, E., Kitayama, Y., Takeuchi, T., 2016. A molecularly imprinted nanocavity-based fluorescence polarization assay platform for cortisol sensing. J. Mater. Chem. B 4, 1770-1777.

Nge, P.N., Rogers, C.I., Woolley, A.T., 2013. Advances in microfluidic materials, functions, integration, and applications. Chem. Rev. 113, 2550-2583.

Niu, H., Yang, Y., Zhang, H., 2015. Efficient one-pot synthesis of hydrophilic and fluorescent molecularly imprinted polymer nanoparticles for direct drug quantification in real biological samples. Biosens. Bioelectron. 74, 440-446.

Novoselov, K.S., Geim, A.K., Morozov, S.V., Jiang, D., Zhang, Y., Dubonos, S.V., Grigorieva, I.V., Firsov, A.A., 2004. Electric field effect in atomically thin carbon films. Science 306, 666-669.

Orozco, J., Cortés, A., Cheng, G., Sattayasamitsathit, S., Gao, W., Feng, X., Shen, Y., Wang, J., 2013. Molecularly imprinted polymer-based catalytic micromotors for selective protein transport. J. Am. Chem. Soc. 135, 5336-5339.

Piletsky, S.A., Piletskaya, E.V., El'Skaya, A.V., Levi, R., Yano, K., Karube, I., 1997. Optical detection system for triazine based on molecularly-imprinted polymers. Anal. Lett. 30, 445-455.

Qi, J., Li, B., Wang, X., Zhang, Z., Wang, Z., Han, J., Chen, L., 2017. Three-dimensional paper-based microfluidic chip device for multiplexed fluorescence detection of $\mathrm{Cu}^{2+}$ and $\mathrm{Hg}^{2+}$ ions based on ion imprinting technology. Sens. Actuators B: Chem. 251, 224-233.

Qian, K., Fang, G., Wang, S., 2013. Highly sensitive and selective novel core-shell molecularly imprinted polymer based on $\mathrm{NaYF}_{4}: \mathrm{Yb}^{3+}, \mathrm{Er}^{3+}$ upconversion fluorescent nanorods. RSC Adv. 3, 3825-3828.

Qiu, C., Xing, Y., Yang, W., Zhou, Z., Wang, Y., Liu, H., Xu, W., 2015. Surface molecular imprinting on hybrid SiO2-coated CdTe nanocrystals for selective optosensing of bisphenol A and its optimal design. Appl. Surf. Sci. 345, 405-417.

Qiu, H., Gao, L., Wang, J., Pan, J., Yan, Y., Zhang, X., 2017. A precise and efficient detection of beta-cyfluthrin via fluorescent molecularly imprinted polymers with ally fluorescein as functional monomer in agricultural products. Food Chem. 217, 620-627.

Rouhani, S., Nahavandifard, F., 2014. Molecular imprinting-based fluorescent optosensor using a polymerizable 1,8-naphthalimide dye as a florescence functional monomer. Sens. Actuators B: Chem. 197, 185-192.

Schrum, K.F., Lancaster, J.M., Johnston, S.E., Gilman, S.D., 2000. Monitoring electroosmotic flow by periodic photobleaching of a dilute, neutral fluorophore. Anal. Chem. 72, 4317-4321.

Shang, L., Dong, S., Nienhaus, G.U., 2011. Ultra-small fluorescent metal nanoclusters: synthesis and biological applications. Nano Today 6, 401-418.

Shinde, S., El-Schich, Z., Malakpour, A., Wan, W., Dizeyi, N., Mohammadi, R., Rurack, K., Wingren, A.G., Sellergren, B., 2015. Sialic acid-imprinted fluorescent core-shell particles for selective labeling of cell surface glycans. J. Am. Chem. Soc. 137, 13908-13912.

Shustova, N.B., Cozzolino, A.F., Reineke, S., Baldo, M., Dincă, M., 2013. Selective turn-on ammonia sensing enabled by high-temperature fluorescence in metal-organic frameworks with open metal sites. J. Am. Chem. Soc. 135, 13326-13329.

Silvi, S., Credi, A., 2015. Luminescent sensors based on quantum dot-molecule 
conjugates. Chem. Soc. Rev. 44, 4275-4289.

Subrahmanyam, S., Piletsky, S.A., Piletska, E.V., Chen, B., Day, R., Turner, A.P.F., 2000. "Bite-and-switch" approach to creatine recognition by use of molecularly imprinted polymers. Adv. Mater. 12, 722-724.

Suga, Y., Sunayama, H., Ooya, T., Takeuchi, T., 2013. Molecularly imprinted polymers prepared using protein-conjugated cleavable monomers followed by site-specific post-imprinting introduction of fluorescent reporter molecules. Chem. Commun. 49, $8450-8452$.

Sun, X., Wang, Y., Lei, Y., 2015. Fluorescence based explosive detection: from mechanisms to sensory materials. Chem. Soc. Rev. 44, 8019-8061.

Sunayama, H., Ooya, T., Takeuchi, T., 2010. Fluorescent protein recognition polymer thin films capable of selective signal transduction of target binding events prepared by molecular imprinting with a post-imprinting treatment. Biosens. Bioelectron. 26, 458-462.

Sunayama, H., Ooyaa, T., Takeuchi, T., 2014. Fluorescent protein-imprinted polymers capable of signal transduction of specific binding events prepared by a site-directed two-step post-imprinting modification. Chem. Commun. 50, 1347-1349.

Sunayama, H., Takeuchi, T., 2014. Molecularly imprinted protein recognition cavities bearing exchangeable binding sites for postimprinting site-directed introduction of reporter molecules for readout of binding events. ACS Appl. Mater. Interfaces 6 20003-20009.

Sunayama, H., Ohta, T., Kuwahara, A., Takeuchi, T., 2016. Fluorescence signaling molecularly imprinted polymers for antibiotics prepared via site-directed post-imprinting introduction of plural fluorescent reporters within the recognition cavity. J. Mater. Chem. B 4, 7138-7145.

Takeuchi, T., Sunayama, H., Takano, E., Kitayama, Y., 2015. Post-imprinting and incavity functionalization. Adv. Biochem. Eng. Biotechnol. 150, 95-106.

Tang, Y., Gao, Z., Wang, S., Gao, X., Gao, J., Ma, Y., Liu, X., Li, J., 2015. Upconversion particles coated with molecularly imprinted polymers as fluorescence probe for detection of clenbuterol. Biosens. Bioelectron. 71, 44-50.

Tang, Y., Liu, H., Gao, J., Liu, X., Gao, X., Lu, X., Fang, G., Wang, J., Li, J., 2018. Upconversion particle@ $\mathrm{Fe}_{3} \mathrm{O}_{4} @$ molecularly imprinted polymer with controllable shell thickness as high-performance fluorescent probe for sensing quinolones. Talanta 181, 95-103.

Ton, X.A., Acha, V., Haupt, K., Bui, B.T.S., 2012. Direct fluorimetric sensing of UV-excited analytes in biological and environmental samples using molecularly imprinted polymer nanoparticles and fluorescence polarization. Biosens. Bioelectron. 36, 22-28.

Udomsap, D., Branger, C., Culioli, G., Dollet, P., Brisset, H., 2014. A versatile electrochemical sensing receptor based on a molecularly imprinted polymer. Chem. Commun. 50, 7488-7491.

Urraca, J.L., Barrios, C.A., Canalejas-Tejero, V., Orellana, G., Moreno-Bondi, M.C., 2014. Molecular recognition with nanostructures fabricated by photopolymerization within metallic subwavelength apertures. Nanoscale 6, 8656-8663.

Valeur, B., Leray, I., 2000. Design principles of fluorescent molecular sensors for cation recognition. Coord. Chem. Rev. 205, 3-40.

Verma, A., Uzun, O., Hu, Y., Hu, Y., Han, H.S., Watson, N., Chen, S., Irvine, D.J., Stellacci, F., 2008. Surface-structure-regulated cell-membrane penetration by monolayer-protected nanoparticles. Nat. Mater. 7, 588-595.

Wan, W., Biyikal, M., Wagner, R., Sellergren, B., Rurack, K., 2013. Fluorescent sensory microparticles that "light-up" consisting of a silica core and a molecularly imprinted polymer (MIP) shell. Angew. Chem. Int. Ed. 52, 7023-7027.

Wang, X., Li, Y., Wang, H., Fu, Q., Peng, J., Wang, Y., Du, J., Zhou, Y., Zhan, L., 2015. Gold nanorod-based localized surface plasmon resonance biosensor for sensitive detection of hepatitis B virus in buffer, blood serum and plasma. Biosens. Bioelectron. 64, 404-410.

Wang, X., Yu, J., Wu, X., Fu, J., Kang, Q., Shen, D., Li, J., Chen, L., 2016b. A molecular imprinting-based turn-on Ratiometric fluorescence sensor for highly selective and sensitive detection of 2,4-dichlorophenoxyacetic acid (2,4-D). Biosens. Bioelectron. 81, 438-444.

Wang, X., Yu, J., Kang, Q., Shen, D., Li, J., Chen, L., 2016a. Molecular imprinting ratiometric fluorescence sensor for highly selective and sensitives detection of phycocyanin. Biosens. Bioelectron. 77, 624-630.

Wang, J., Qiu, H., Shen, H., Pan, J., Dai, X., Yan, Y., Pan, G., Sellergren, B., 2016c. Molecularly imprinted fluorescent hollow nanoparticles as sensors for rapid and efficient detection $\lambda$-cyhalothrin in environmental water. Biosens. Bioelectron. 85, 387-394.

Wang, X., Yu, J., Li, J., Kang, Q., Shen, D., Chen, L., 2018a. Quantum dots based imprinting fluorescent nanosensor for the selective and sensitive detection of phycocyanin: a general imprinting strategy toward proteins. Sens. Actuators B: Chem. 255, 268-274.

Wang, X., Yu, S., Liu, W., Fu, L., Wang, Y., Li, J., Chen, L., 2018b. Molecular imprinting based hybrid ratiometric fluorescence sensor for the visual determination of bovine hemoglobin. ACS Sens. 3, 378-385.

Wang, Y., Hu, A., 2014. Carbon quantum dots: synthesis, properties and applications. J. Mater. Chem. C 2, 6921-6939.

Wagner, R., Wan, W., Biyikal, M., Benito-Peña, E., Moreno-Bondi, M.C., Lazraq, I., Rurack, K., Sellergren, B., 2013. Synthesis, spectroscopic, and analyte-responsive behavior of a polymerizable naphthalimide-based carboxylate probe and molecularly imprinted polymers prepared thereof. J. Org. Chem. 78, 1377-1389.

Ware, W.R., 1962. Oxygen quenching of fluorescence in solution: an experimental study of the diffusion process. J. Phys. Chem. 66, 455-458.

Wei, X., Zhou, Z., Hao, T., Li, H., Zhu, Y., Gao, L., Yan, Y., 2015. A novel molecularly imprinted polymer thin film at surface of $\mathrm{ZnO}$ nanorods for selective fluorescence detection of para-nitrophenol. RSC Adv. 5, 44088-44095.
Wei, X., Xu, G., Gong, C., Qin, F., Gong, X., Li, C., 2018. Fabrication and evaluation of sulfanilamide-imprinted composite sensors by developing a custom-tailored strategy. Sens. Actuators B: Chem. 255, 2697-2703.

Wu, Y., Liu, Y., Gao, X., Gao, K., Xia, H., Luo, M., Wang, X., Ye, L., Shi, Y., Lu, B., 2015a Monitoring bisphenol A and its biodegradation in water using a fluorescent molecularly imprinted chemosensor. Chemosphere 119, 515-523.

Wu, X., Zhang, Z., Li, J., You, H., Li, Y., Chen, L., 2015b. Molecularly imprinted polymerscoated gold nanoclusters for fluorescent detection of bisphenol A. Sens. Actuators B: Chem. 211, 507-514.

Wu, Z., Li, J., Ávila, B.E.F., Li, T., Gao, W., He, Q., Zhang, L., Wang, J., 2015c. Waterpowered cell-mimicking janus micromotor. Adv. Funct. Mater. 25, 7497-7501.

Xu, S., Lu, H., Li, J., Song, X., Wang, A., Chen, L., Han, S., 2013. Dummy molecularly imprinted polymers-capped CdTe quantum dots for the fluorescent sensing of 2,4,6trinitrotoluene. ACS Appl. Mater. Interfaces 5, 8146-8154.

Xu, S., Lu, H., 2015a. One-pot synthesis of mesoporous structured ratiometric fluorescence molecularly imprinted sensor for highly sensitive detection of melamine from milk samples. Biosens. Bioelectron. 73, 160-166.

Xu, S., Lu, H., 2015b. Ratiometric fluorescence and mesoporous structure dual signal amplification for sensitive and selective detection of TNT based on MIP@QD fluorescence sensors. Chem. Commun. 51, 3200-3203.

Xu, S., Lu, H., 2016. Mesoporous structured MIPs@CDs fluorescence sensor for highly sensitive detection of TNT. Biosens. Bioelectron. 85, 950-956.

Xu, X., Ray, R., Gu, Y., Ploehn, H.J., Gearheart, L., Raker, K., Scrivens, W.A., 2004. Electrophoretic analysis and purification of fluorescent single-walled carbon nanotube fragments. J. Am. Chem. Soc. 126, 12736-12737.

Xu, Z., Deng, P., Li, J., Xu, L., Tang, S., 2017. Molecularly imprinted fluorescent probe based on FRET for selective and sensitive detection of doxorubicin. Mat. Sci. Eng. BAdv. 218, 31-39.

Yan, Y.J., He, X.W., Li, W.Y., Zhang, Y.K., 2017. Nitrogen-doped graphene quantum dotslabeled epitope imprinted polymer with double templates via the metal chelation for specific recognition of cytochrome c. Biosens. Bioelectron. 91, 253-261.

Yang, Q., Peng, H., Li, J., Li, Y., Xiong, H., Chen, L., 2017a. Label-free colorimetric detection of tetracycline using analyte-responsive inverse-opal hydrogels based on molecular imprinting technology. New J. Chem. 41, 10174-10180.

Yang, J., Lin, Z.Z., Zhong, H.P., Chen, X.M., Huang, Z.Y., 2017b. Determination of leucomalachite green in fish using a novel MIP-coated QDs probe based on synchronous fluorescence quenching effect. Sens. Actuators B: Chem. 252, 561-567.

Yang, Q., Wu, X., Peng, H., Fu, L., Song, X., Li, J., Xiong, H., Chen, L., 2018. Simultaneous phase-inversion and imprinting based sensor for highly sensitive and selective detection of bisphenol A. Talanta 176, 595-603.

Yang, Y., Wang, Z., Niu, H., Zhang, H., 2016. One-pot synthesis of quantum dot-labeled hydrophilic molecularly imprinted polymer nanoparticles for direct optosensing of folic acid in real, undiluted biological samples. Biosens. Bioelectron. 86, 580-587.

Yong, K.T., Law, W.C., Hu, R., Ye, L., Liu, L., Swihart, M.T., Prasad, P.N., 2013. Nanotoxicity assessment of quantum dots: from cellular to primate studies. Chem. Soc. Rev. 42, 1236-1250.

Yoo, J.M., Kang, J.H., Hong, B.H., 2015. Graphene-based nanomaterials for versatile imaging studies. Chem. Soc. Rev. 44, 4835-4852.

Yu, J., Wang, X., Kang, Q., Li, J., Shen, D., Chen, L., 2017. One-pot synthesis of a quantum dot-based molecular imprinting nanosensor for highly selective and sensitive fluorescence detection of 4-nitrophenol in environmental waters. Environ. Sci.: Nano 4, 493-502.

Zhang, L.M., Huang, X.Y., Zhang, Y.M., Li, J.P., Zhang, L., Zeng, Y., 2016. A novel molecularly imprinted fluorescence test strip for detection of cimaterol. Chin. J. Anal. Chem. 44, 1477-1481.

Zhang, X., Yang, S., Jiang, R., Sun, L., Pang, S., Luo, A., 2018. Fluorescent molecularly imprinted membranes as biosensor for the detection of target protein. Sens. Actuators B: Chem. 254, 1078-1086.

Zhang, Z., Li, J., Wang, X., Shen, D., Chen, L., 2015a. Quantum dots based mesoporous structured imprinting microspheres for the sensitive fluorescent detection of phycocyanin. ACS Appl. Mater. Interfaces 7, 9118-9127.

Zhang, Z., Li, J., Fu, L., Liu, D., Chen, L., 2015b. Magnetic molecularly imprinted microsensor for selective recognition and transport of fluorescent phycocyanin in seawater. J. Mater. Chem. A 3, 7437-7444.

Zhao, T., Wang, J., He, J., Deng, Q., Wang, S., 2017. One-step post-imprint modification achieve dual-function of glycoprotein fluorescent sensor by "Click Chemistry". Biosens. Bioelectron. 91, 756-761.

Zheng, J., Nicovich, P.R., Dickson, R.M., 2007. Highly fluorescent noble-metal quantum dots. Ann. Rev. Phys. Chem. 58, 409-431.

Zheng, X., Pan, J., Gao, L., Wei, X., Dai, J.D., Shi, W.D., Yan, Y., 2015. Silica nanoparticles doped with a europium(III) complex and coated with an ion imprinted polymer for rapid determination of copper(II). Microchim. Acta 182, 753-761.

Zhou, J., Yang, Y., Zhang, C., 2015a. Toward biocompatible semiconductor quantum dots: from biosynthesis and bioconjugation to biomedical application. Chem. Rev. 115, 11669-11717.

Zhou, X., Wang, A., Yu, C., Wu, S., Shen, J., 2015b. Facile synthesis of molecularly imprinted graphene quantum dots for the determination of dopamine with affinityadjustable. ACS Appl. Mater. Interfaces 7, 11741-11747.

Zhou, Y., Qu, Z., Zeng, Y., Zhou, T., Shi, G., 2014. A novel composite of graphene quantum dots and molecularly imprinted polymer for fluorescent detection of paranitrophenol. Biosens. Bioelectron. 52, 317-323.

Zor, E., Morales-Narváez, E., Zamora-Gálvez, A., Bingol, H., Ersoz, M., Merkoçi, A., 2015. Graphene quantum dots-based photoluminescent sensor: a multifunctional composite for pesticide detection. ACS Appl. Mater. Interfaces 7, 20272-20279. 